\title{
Search for a Heavy Particle Decaying into an Electron and a Muon with the ATLAS Detector in $\sqrt{s}=7 \mathrm{TeV} p p$ collisions at the $\mathrm{LHC}$
}

\author{
G. Aad et al.* \\ (ATLAS Collaboration) \\ (Received 29 March 2011; published 22 June 2011)
}

\begin{abstract}
This Letter presents the first search for a heavy particle decaying into an $e^{ \pm} \mu^{\mp}$ final state in $\sqrt{s}=$ $7 \mathrm{TeV} p p$ collisions at the LHC. The data were recorded by the ATLAS detector during 2010 and correspond to a total integrated luminosity of $35 \mathrm{pb}^{-1}$. No excess above the standard model background expectation is observed. Exclusions at $95 \%$ confidence level are placed on two representative models. In an $R$-parity violating supersymmetric model, tau sneutrinos with a mass below $0.75 \mathrm{TeV}$ are excluded, assuming all $R$-parity violating couplings are zero except $\lambda_{311}^{\prime}=0.11$ and $\lambda_{312}=0.07$. In a lepton flavor violating model, a $Z^{\prime}$-like vector boson with masses of $0.70-1.00 \mathrm{TeV}$ and corresponding cross sections times branching ratios of $0.175-0.183 \mathrm{pb}$ is excluded. These results extend to higher mass $R$-parity violating sneutrinos and lepton flavor violating $Z$ 's than previous constraints from the Tevatron.
\end{abstract}

PACS numbers: 12.60.Jv, 12.60.Cn, 13.85.Rm, 14.80.Ly

Events with $e^{ \pm} \mu^{\mp}(e \mu)$ in the final state, which played an important role in the discoveries of the tau lepton and the top quark, have a clean experimental signature and low background. Many new physics models allow an $e \mu$ signature. For example, in $R$-parity violating (RPV) supersymmetric models [1] a sneutrino can decay to $e \mu$. Models with additional gauge symmetry can accommodate an $e \mu$ signature through lepton flavor violating (LFV) decays of an extra gauge boson $Z^{\prime}$ [2]. Standard model (SM) processes that can produce an $e \mu$ signature typically have small cross sections, and the $e \mu$ invariant mass $\left(m_{e \mu}\right)$ lies below the range favored for new physics signals. This Letter reports a search for a heavy particle decaying into the $e \mu$ final state using data taken with the ATLAS detector. The results are interpreted in terms of the production and decay of a tau sneutrino $\tilde{\nu}_{\tau}$ and a $Z^{\prime}$. Both the CDF and D0 Collaborations at the Tevatron collider have reported searches for the RPV production and decay of the $\tilde{\nu}_{\tau}$ [3-7]. The CDF Collaboration has also set limits on the LFV couplings as a function of the $Z^{\prime}$ mass [4].

The ATLAS detector [8] is a multipurpose particle physics apparatus with a forward-backward symmetric cylindrical geometry and near $4 \pi$ coverage in solid angle [9]. The inner tracking detector (ID) consists of a silicon pixel detector, a silicon microstrip detector, and a transition radiation tracker. The ID is surrounded by a thin superconducting solenoid providing a $2 \mathrm{~T}$ magnetic field and by a finely segmented, hermetic calorimeter. The latter covers $|\eta|<4.9$ and provides three-dimensional reconstruction

*Full author list given at the end of the article.

Published by the American Physical Society under the terms of the Creative Commons Attribution 3.0 License. Further distribution of this work must maintain attribution to the author(s) and the published article's title, journal citation, and DOI. of particle showers using lead-liquid argon sampling for the electromagnetic compartment followed by a hadronic compartment which is based on iron-scintillating tiles sampling in the central region and on liquid argon sampling with copper or tungsten absorbers for $|\eta|>1.7$. The muon spectrometer surrounds the calorimeters and consists of three large superconducting toroids, a system of precision tracking chambers, and detectors for triggering.

The data used in this analysis were recorded in 2010 at a center-of-mass energy $\sqrt{s}=7 \mathrm{TeV}$. Application of dataquality requirements results in a total integrated luminosity of $35 \mathrm{pb}^{-1}$ with an estimated uncertainty of $11 \%$ [10]. Events are required to satisfy one of the single lepton ( $e$ or $\mu$ ) triggers, which have nominal transverse momentum $p_{T}$ thresholds up to $15 \mathrm{GeV}$ for $e$ and $13 \mathrm{GeV}$ for $\mu$. The trigger efficiency is measured to be $100 \%$, with a precision of $1 \%$, for $e \mu$ candidates containing two leptons with transverse momentum $p_{T}>20 \mathrm{GeV}$.

To select $e \mu$ events, the electron candidate is required to have $p_{T}>20 \mathrm{GeV}$ and to lie inside the pseudorapidity regions $|\eta|<1.37$ or $1.52<|\eta|<2.47$. The event is rejected if the candidate cluster is located in a problematic region of the electromagnetic calorimeter. Electron identification and isolation requirements provide rejection against hadrons. A set of electron identification criteria based on the calorimeter shower shape, track quality, and track matching with the calorimeter cluster, referred to as "medium" in Ref. [11], is applied. In addition, a calorimeter isolation criterion $E_{T}^{\Delta R<0.2} / E_{T}<0.1$ is applied, where $E_{T}^{\Delta R<0.2}$ is defined as the transverse energy deposited in the calorimeter within a cone of radius $\Delta R=\sqrt{\Delta \eta^{2}+\Delta \phi^{2}}<$ 0.2 around the electron cluster, excluding the core energy deposited by the electron, and $E_{T}$ is the transverse energy of the electron.

The muon candidate must be reconstructed in both the ID and the muon spectrometer. A good match of the 
parameters of the ID and muon spectrometer tracks is required, and the $p_{T}$ values measured by these two systems must be compatible. Furthermore, the muon candidate must have $p_{T}>20 \mathrm{GeV},|\eta|<2.4$, and be isolated in the ID with $p_{T}^{\Delta R<0.2} / p_{T}<0.1$, where $p_{T}^{\Delta R<0.2}$ is defined as the sum of the $p_{T}$ of tracks with $p_{T}>1 \mathrm{GeV}$ within a cone of radius $\Delta R<0.2$ around the muon track, excluding the muon track.

Jets are reconstructed by using the anti- $k_{t}$ jet clustering algorithm [12] with a radius parameter of 0.4. Only jets with $p_{T}>20 \mathrm{GeV}$ and $|\eta|<2.5$ are considered. If such a jet and an electron lie within $\Delta R=0.2$ of each other, the jet is discarded. Leptons are considered only if they are separated from all of the remaining jets by $\Delta R>0.4$. Electrons and muons are also required to be separated from each other by $\Delta R>0.2$.

The $e \mu$ candidate events are required to have exactly one electron and one muon with opposite charge satisfying the above selection criteria. Furthermore, events have to contain at least one primary vertex reconstructed with more than four associated tracks with $p_{T}>150 \mathrm{MeV}$.

The SM processes that can produce an $e \mu$ signature are predominantly $t \bar{t}, Z / \gamma^{*} \rightarrow \tau \tau, W / Z+$ jets, diboson, single top, and QCD multijet events. Among the processes listed above, $t \bar{t}, Z / \gamma^{*} \rightarrow \tau \tau$, single top, $W W, W Z$, and $Z Z$ produce electrons and muons in the final state and amount to $\sim 80 \%$ of the expected $e \mu$ data yield. The contributions from these processes are estimated by using Monte Carlo (MC) samples generated at $\sqrt{s}=7 \mathrm{TeV}$ and processed with the standard chain of the ATLAS GEANT4 [13] simulation and reconstruction [14] using the ATLAS MC09 parameter tune [15]. The event generators used are PYTHIA 6.421 [16] ( $W$ and $\left.Z / \gamma^{*}\right)$, POWHEG 1.0 [17] $(t \bar{t})$, MADGRAPH 4 [18] $(W / Z+\gamma)$, MC@NLO 3.4 [19] (single top), and HERWIG $6.510[20]$ ( $W W, W Z$, and $Z Z$ ). The MC samples are normalized to cross sections with higher order corrections applied, as follows. The cross section is calculated to next-to-next-to-leading-order accuracy for $W$ and $Z / \gamma^{*}$ [21], next-to-leading-order plus next-to-next-to-leading-log for $t \bar{t}$ [22], and next-to-leading-order for $W W, W Z$, and $Z Z$ [23]. Single top and $W / Z+\gamma$ cross sections come from MC@NLO and MADGRAPH, respectively. Studies of $Z / \gamma^{*} \rightarrow \ell \ell(\ell=e, \mu)$ events have shown that the lepton reconstruction and identification efficiencies, energy scale, and resolution need to be adjusted in the MC calculations to properly describe the data. The appropriate corrections are applied to the $\mathrm{MC}$ calculations in order to improve the modeling of the backgrounds.

The processes $W / Z+\gamma, W / Z+$ jets, and pure QCD jet production give rise to background in addition to prompt leptons, which come from $W$ and $Z$ decay. Jets misidentified as leptons, electrons from photon conversions, and leptons from hadron decays (including $b$ - and $c$-hadron decays) are classified as instrumental background. The instrumental background accounts for $\sim 20 \%$ of the expected $e \mu$ data yield. The dominant component of the instrumental background comes from events with one prompt lepton and one jet identified as a lepton, with an additional contribution from events with two misidentified jets. These sources are referred to as jet instrumental background and are estimated by using data. The background component initiated by prompt photons, referred to as the photon instrumental background, is estimated from MC calculations.

The jet instrumental background is estimated by using data in the following way. Two selections are defined, both requiring at least one lepton satisfying all lepton criteria. The selections are defined by the second lepton candidate: The "tight" selection requires the second candidate to pass all lepton criteria, while the "loose" selection does not enforce the lepton isolation requirement. The probability for a lepton to satisfy the lepton isolation requirement is estimated by applying the loose and tight selections on $Z / \gamma^{*} \rightarrow \ell \ell$ events. The probability for a jet to satisfy the lepton isolation requirement is estimated by applying the loose and tight selections on a sample of QCD dijet events to which a cut on the missing transverse energy $E_{T}^{\text {miss }}<$ $15 \mathrm{GeV}$ is applied to remove $W+$ jets events. These two probabilities, along with numbers of events selected in the loose and tight samples, are used to estimate the background in the final selected sample. The background is estimated to be $12_{-5}^{+10}$ events for candidates with one electron and one jet satisfying the muon selection criteria and $19_{-7}^{+28}$ events for candidates with one muon and one jet satisfying the electron selection criteria. The dominant uncertainty on the jet instrumental background estimation comes from the $p_{T}$ dependence of the probability for a jet to pass the lepton isolation criterion. The background due to two jets satisfying the lepton requirements is estimated to be $1.3_{-1.3}^{+5}$ events from same-sign dilepton events in the data with the subtraction of contributions estimated from MC calculations for all physics processes except the QCD multijet. The overall jet instrumental background is found to be $29_{-10}^{+30}$ events. This background level has been checked in simulated samples, which agree with the datadriven estimates.

The dominant photon instrumental background comes from the $W(\rightarrow \mu \nu) \gamma$ and $Z(\rightarrow \mu \mu) \gamma$ processes where the photon is reconstructed as an electron. A photon can be reconstructed as an electron if it lies close to a charged particle track or the photon converts to $e^{+} e^{-}$after interacting with the material in front of the calorimeter. The photon instrumental background is found to be $4.0 \pm 0.7$ events.

Table I shows the number of events selected in the data and the estimated background contributions with their uncertainties. A total of $160 e \mu$ candidates are observed in the data, while the expectation from SM processes is $163_{-18}^{+34}$ events. The dominant sources of systematic uncertainty for the SM prediction arise from the uncertainty on 
TABLE I. Estimated backgrounds in the selected sample, together with the observed event yield.

\begin{tabular}{lc}
\hline \hline Process & Number of events \\
\hline$Z / \gamma^{*} \rightarrow \tau \tau$ & $54 \pm 7$ \\
$t \bar{t}$ & $57 \pm 9$ \\
$W W$ & $13.4 \pm 1.7$ \\
Single top & $4.6 \pm 0.9$ \\
$W Z$ & $0.79 \pm 0.11$ \\
Instrumental background & $33_{-10}^{+30}$ \\
Total background & $163_{-18}^{+34}$ \\
Data & 160 \\
\hline \hline
\end{tabular}

the probability for a jet to satisfy the lepton isolation requirement (70\% for an electron and 30\% for a muon), theoretical cross sections on the physics background processes $(5 \%-15 \%)$, and the integrated luminosity $(11 \%)$. Other systematic uncertainties from the lepton trigger, reconstruction and identification efficiencies, energy or momentum scale, and resolution have been included and are small.

Since no excess is observed in the data, limits are set for the production of $\tilde{\nu}_{\tau}$ in RPV supersymmetric models and an LFV $Z^{\prime}$-like vector boson.

The RPV production of $\tilde{\nu}_{\tau}$ by $d \bar{d}$ annihilation decaying into $e \mu$ is considered. By fixing all RPV couplings but $\lambda_{311}^{\prime}$ and $\lambda_{312}$ to zero, the contributions to the $e \mu$ final state originate from the $\tilde{\nu}_{\tau}$ only. With these couplings, along with the assumption that $\tilde{\nu}_{\tau}$ is the lightest supersymmetric particle, the $\tilde{\nu}_{\tau}$ can decay only to $d \bar{d}$ or $e \mu$. The signal cross section depends on the $\tilde{\nu}_{\tau}$ mass $\left(m_{\tilde{\nu}_{\tau}}\right), \lambda_{311}^{\prime}$, and $\lambda_{312}$. The third-generation $\tilde{\nu}_{\tau}$ is considered since stringent limits exist on the electron sneutrino and muon sneutrino [1]. The couplings $\lambda_{311}^{\prime}=0.11$ and $\lambda_{312}=0.07$, compatible with the current indirect limits [1], are chosen as a benchmark point.

An $e \mu$ resonance can be generated in models containing a heavy neutral gauge boson with nondiagonal lepton flavor couplings $Z^{\prime}$ [24]. Very stringent limits on the combination of the mass and the coupling to $e e$ and $e \mu$ of such models have been inferred from searches for rare muon decay [2]. By using the data presented in this Letter, a limit on the production cross section times branching ratio to $e \mu$ can be placed on a $Z$-like vector boson. To calculate the acceptance and efficiency, the $Z^{\prime}$ is assumed to have the same quark and lepton couplings as the SM $Z$.

MC events with $\tilde{\nu}_{\tau}$ or $Z^{\prime}$ decaying into $e \mu$ are generated with HERWIG [20,25] or PYTHIA, respectively. Samples are produced with sneutrino masses ranging from 0.1 to $1 \mathrm{TeV}$ and $Z^{\prime}$ masses from 0.7 to $1 \mathrm{TeV}$.

The $e \mu$ invariant mass distribution is presented in Fig. 1 for data, background contributions, and two possible new physics signals: a $\tilde{\nu}_{\tau}$ with $m_{\tilde{\nu}_{\tau}}=650 \mathrm{GeV}$ and a $Z^{\prime}$ with $m_{Z^{\prime}}=700 \mathrm{GeV}$. The cross section is $0.31 \mathrm{pb}$ for $m_{\tilde{\nu}_{\tau}}=$ $650 \mathrm{GeV}$ [26] and $0.61 \mathrm{pb}$ for $m_{Z^{\prime}}=700 \mathrm{GeV}$ [27]. The

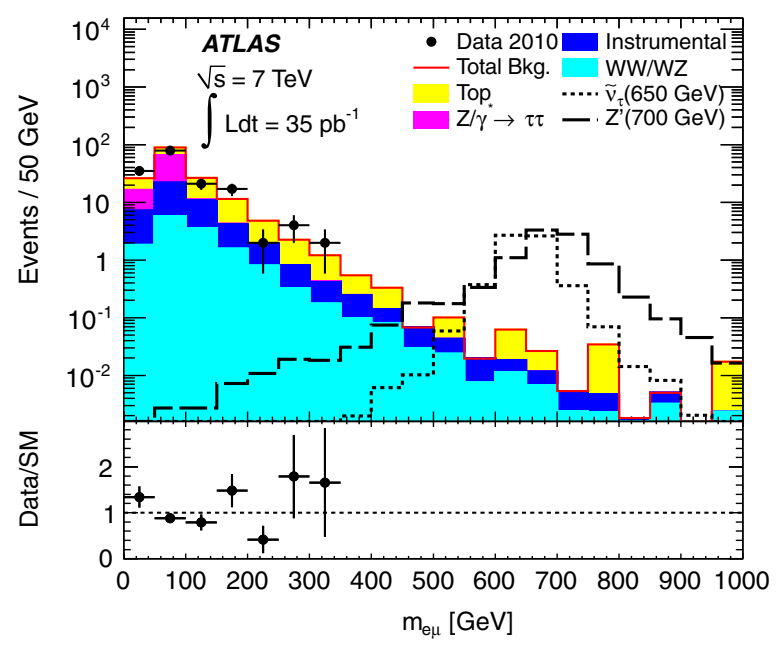

FIG. 1 (color online). Observed and predicted $e \mu$ invariant mass distributions. Signal simulations are shown for $m_{\tilde{\nu}_{\tau}}=$ $650 \mathrm{GeV}\left(\lambda_{311}^{\prime}=0.11\right.$ and $\left.\lambda_{312}=0.07\right)$ and $m_{Z^{\prime}}=700 \mathrm{GeV}$. The ratio plot at the bottom includes only statistical uncertainties.

corresponding overall acceptance times efficiency is 55\% for $\tilde{\nu}_{\tau}$ and $50 \%$ for $Z^{\prime}$.

The $m_{e \mu}$ spectrum is examined for the presence of a new heavy particle. For $m_{\tilde{\nu}_{\tau}}<500 \mathrm{GeV}$, the search region for specific $m_{\tilde{\nu}_{\tau}}$ is defined to be $\left(m_{\tilde{\nu}_{\tau}}-3 \sigma, m_{\tilde{\nu}_{\tau}}+3 \sigma\right)$, where $\sigma$ is the expected $m_{e \mu}$ resolution (e.g., $\sigma \simeq 15 \mathrm{GeV}$ for $\left.m_{\tilde{\nu}_{\tau}}=400 \mathrm{GeV}\right)$. For higher $m_{\tilde{\nu}_{\tau}}$, the region $m_{e \mu}>$ $400 \mathrm{GeV}$ is used. The expected and observed $95 \%$ C.L. upper limits on $\sigma\left(p p \rightarrow \tilde{\nu}_{\tau}\right) \times \mathrm{BR}\left(\tilde{\nu}_{\tau} \rightarrow e \mu\right)$ are calculated by using a Bayesian method [28] with a flat prior for the signal cross section as a function of $m_{\tilde{\nu}_{\tau}}$. Figure 2(a) shows the expected and observed limits, as a function of $m_{\tilde{\nu}_{\tau}}$, together with the \pm 1 and \pm 2 standard deviation uncertainty bands. The expected exclusion limits are determined by using simulated pseudoexperiments containing only SM processes by evaluating the $95 \%$ C.L. upper limits for each pseudoexperiment at each value of $m_{\tilde{\nu}_{\tau}}$. The median of the distribution of limits is shown as the expected limit. The ensemble of limits is also used to find the $1 \sigma$ and $2 \sigma$ envelope of the expected limits as a function of $m_{\tilde{\nu}_{\tau}}$. For a sneutrino with a mass of $100 \mathrm{GeV}(1 \mathrm{TeV})$, the limit on the cross section times branching ratio is 0.951 (0.154) pb. The theoretical cross sections for $\lambda_{311}^{\prime}=0.11$, $\lambda_{312}=0.07$ and $\lambda_{311}^{\prime}=0.10, \lambda_{312}=0.05$ are also shown. Sneutrinos with masses below $0.75(0.65) \mathrm{TeV}$ are excluded by using $\lambda_{311}^{\prime}=0.11$ and $\lambda_{312}=0.07\left(\lambda_{311}^{\prime}=0.10\right.$ and $\left.\lambda_{312}=0.05\right)$. The results improve on the previous $\mathrm{CDF}$ 95\% C.L. limit of $0.56 \mathrm{TeV}$ assuming $\lambda_{311}^{\prime}=0.10$ and $\lambda_{312}=0.05$. The $95 \%$ C.L. observed upper limits on $\lambda_{311}^{\prime}$ as a function of $m_{\tilde{\nu}_{\tau}}$ are shown in Fig. 2(b) for three values of $\lambda_{312}$, together with the exclusion region obtained from the D0 experiment [7]. The limits derived here are extended to a higher mass region than was available at D0, 

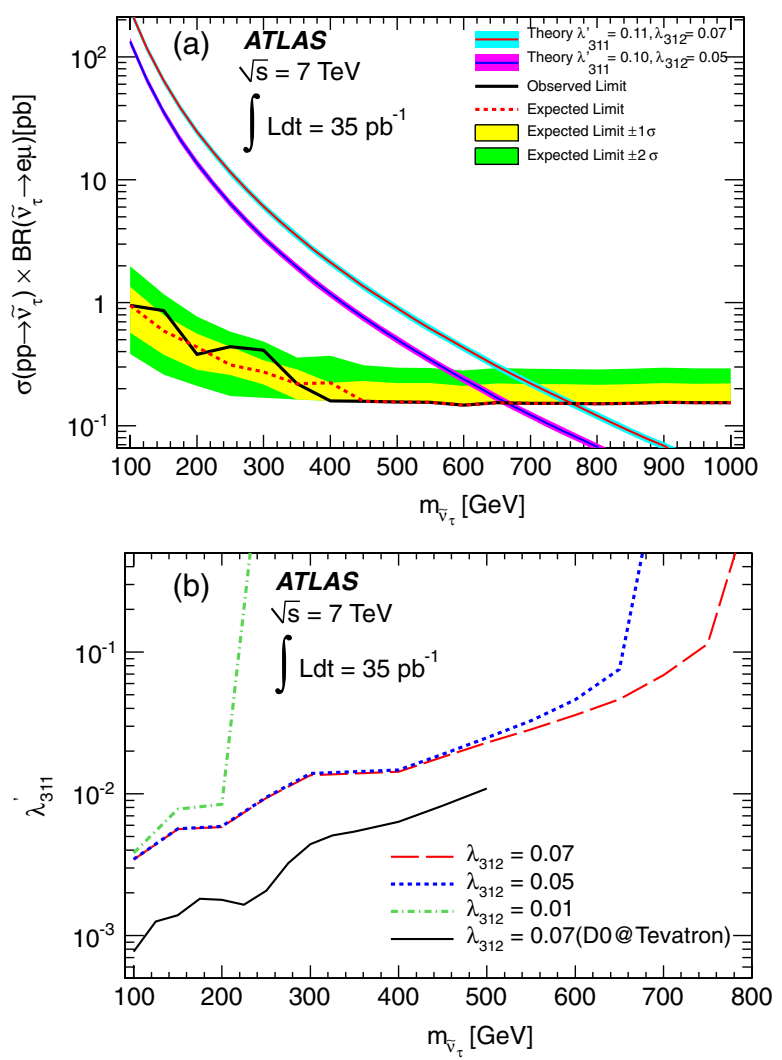

FIG. 2 (color online). (a) The observed 95\% C.L. upper limits on $\sigma\left(p p \rightarrow \tilde{\nu}_{\tau}\right) \times \operatorname{BR}\left(\tilde{\nu}_{\tau} \rightarrow e \mu\right)$ as a function of $m_{\tilde{\nu}_{\tau}}$. The expected limits are also shown together with the \pm 1 and \pm 2 standard deviation uncertainty bands. The theoretical cross sections for $\lambda_{311}^{\prime}=0.11, \lambda_{312}=0.07$ and $\lambda_{311}^{\prime}=0.10, \lambda_{312}=0.05$ are also shown. For $m_{e \mu}>400 \mathrm{GeV}$, the total SM background is $0.67 \pm 0.19$, and the median of number of events expected from pseudoexperiments is 0 ; therefore, the $-1 \sigma$ and $-2 \sigma$ uncertainty bands cannot reach below this expectation. (b) The $95 \%$ C.L. upper limits on the $\lambda_{311}^{\prime}$ coupling as a function of $m_{\tilde{\nu}_{\tau}}$ for three values of $\lambda_{312}$. Regions above the three curves represent ranges of $\lambda_{311}^{\prime}$ values that are excluded. These results are compared to the exclusion region obtained from the D0 experiment.

even though the limits are worse than those obtained by the D0 Collaboration in the low mass region due to limited statistics.

A similar method is used to set limits on the LFV $Z^{\prime}$-like vector boson, by using only events with $m_{e \mu}>400 \mathrm{GeV}$. After finding no events in the data, the $95 \%$ C.L. upper limits on $\sigma\left(p p \rightarrow Z^{\prime}\right) \times \mathrm{BR}\left(Z^{\prime} \rightarrow e \mu\right)$ are set, as shown in Fig. 3. The expected limit is the same as the observed limit because the median background event count expectation is also zero. For a $Z^{\prime}$ with a mass of $700 \mathrm{GeV}(1 \mathrm{TeV})$, the limit on the cross section times branching ratio is 0.175 (0.183) pb. This result improves upon previous CDF limits by probing a higher mass range of $Z^{\prime}$-like vector particles.

In conclusion, a search has been performed for a heavy particle decaying into the $e^{ \pm} \mu^{\mp}$ final state by using $p p$

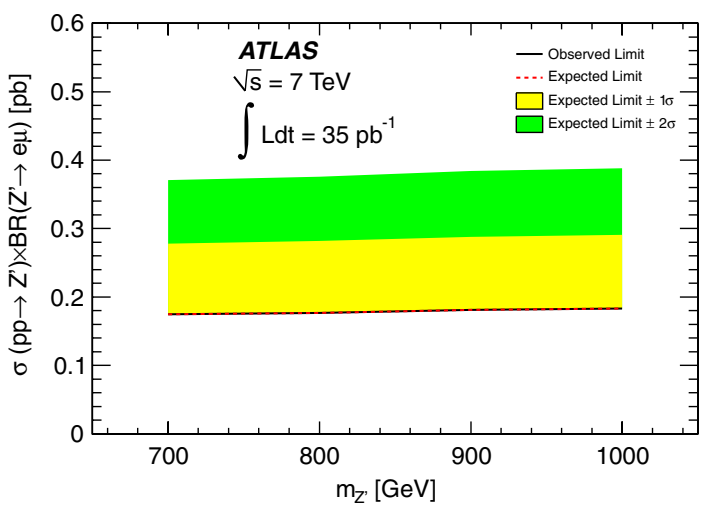

FIG. 3 (color online). The observed 95\% C.L. upper limits on $\sigma\left(p p \rightarrow Z^{\prime}\right) \times \mathrm{BR}\left(Z^{\prime} \rightarrow e \mu\right)$. The expected limits are also shown together with the \pm 1 and \pm 2 standard deviation uncertainty bands. The $-1 \sigma$ and $-2 \sigma$ uncertainty bands cannot reach below the expectation due to the same reason as in Fig. 2(a).

collision data at $\sqrt{s}=7 \mathrm{TeV}$ recorded by the ATLAS detector. The data are found to be consistent with the SM prediction. Exclusions are placed on two representative models at 95\% C.L. In an RPV supersymmetric model, tau sneutrinos with a mass below $0.75 \mathrm{TeV}$ are excluded, assuming single coupling dominance and coupling values $\lambda_{311}^{\prime}=0.11$ and $\lambda_{312}=0.07$. Higher values of the RPV coupling are also excluded as a function of $m_{\tilde{\nu}_{\tau}}$. In an LFV model, extra $Z^{\prime}$-like gauge bosons are excluded with a cross section times branching ratio above $0.183 \mathrm{pb}$, assuming $m_{Z^{\prime}}=1 \mathrm{TeV}$. These results extend to higher mass RPV sneutrinos and LFV Z's than previous constraints from the Tevatron.

We thank CERN for the very successful operation of the LHC, as well as the support staff from our institutions without whom ATLAS could not be operated efficiently. We acknowledge the support of ANPCyT, Argentina; YerPhI, Armenia; ARC, Australia; BMWF, Austria; ANAS, Azerbaijan; SSTC, Belarus; CNPq and FAPESP, Brazil; NSERC, NRC, and CFI, Canada; CERN; CONICYT, Chile; CAS, MOST, and NSFC, China; COLCIENCIAS, Colombia; MSMT CR, MPO CR, and VSC CR, Czech Republic; DNRF, DNSRC, and Lundbeck Foundation, Denmark; ARTEMIS, European Union; IN2P3-CNRS, CEA-DSM/IRFU, France; GNAS, Georgia; BMBF, DFG, HGF, MPG, and AvH Foundation, Germany; GSRT, Greece; ISF, MINERVA, GIF, DIP, and Benoziyo Center, Israel; INFN, Italy; MEXT and JSPS, Japan; CNRST, Morocco; FOM and NWO, The Netherlands; RCN, Norway; MNiSW, Poland; GRICES and FCT, Portugal; MERYS (MECTS), Romania; MES of Russia and ROSATOM, Russian Federation; JINR; MSTD, Serbia; MSSR, Slovakia; ARRS and MVZT, Slovenia; DST/NRF, South Africa; MICINN, Spain; SRC and Wallenberg Foundation, Sweden; SER, SNSF, and Cantons of Bern and Geneva, Switzerland; NSC, Taiwan; TAEK, Turkey; STFC, the Royal Society and Leverhulme 
Trust, United Kingdom; DOE and NSF, United States of America. The crucial computing support from all WLCG partners is acknowledged gratefully, in particular, from CERN and the ATLAS Tier-1 facilities at TRIUMF (Canada), NDGF (Denmark, Norway, Sweden), CCIN2P3 (France), KIT/GridKA (Germany), INFN-CNAF (Italy), NL-T1 (The Netherlands), PIC (Spain), ASGC (Taiwan), RAL (United Kingdom), and BNL (USA) and in the Tier-2 facilities worldwide.

[1] R. Barbier et al., Phys. Rep. 420, 1 (2005).

[2] B. Murakami, Phys. Rev. D 65, 055003 (2002).

[3] D. Acosta et al. (CDF Collaboration), Phys. Rev. Lett. 91, 171602 (2003).

[4] A. Abulencia et al. (CDF Collaboration), Phys. Rev. Lett. 96, 211802 (2006).

[5] T. Aaltonen et al. (CDF Collaboration), Phys. Rev. Lett. 105, 191801 (2010).

[6] V. M. Abazov et al. (D0 Collaboration), Phys. Rev. Lett. 100, 241803 (2008).

[7] V. M. Abazov et al. (D0 Collaboration), Phys. Rev. Lett. 105, 191802 (2010).

[8] G. Aad et al. (ATLAS Collaboration), JINST 3, S08003 (2008).

[9] ATLAS uses a right-handed coordinate system with its origin at the nominal interaction point in the center of the detector and the $z$ axis along the beam pipe. The $x$ axis points from the interaction point to the center of the LHC ring, and the $y$ axis points upward. Cylindrical coordinates $(r, \phi)$ are used in the transverse plane, $\phi$ being the azimuthal angle around the beam pipe. The pseudorapidity is defined in terms of the polar angle $\theta$ as $\eta=$ $-\ln \tan (\theta / 2)$.

[10] G. Aad et al. (ATLAS Collaboration), Eur. Phys. J. C 71, 1630 (2011).
[11] G. Aad et al. (ATLAS Collaboration), J. High Energy Phys. 12 (2010) 060.

[12] M. Cacciari, G. P. Salam, and G. Soyez, J. High Energy Phys. 04 (2008) 063.

[13] S. Agostinelli et al. (GEANT4 Collaboration), Nucl. Instrum. Methods Phys. Res., Sect. A 506, 250 (2003).

[14] G. Aad et al. (ATLAS Collaboration), Eur. Phys. J. C 70, 823 (2010).

[15] ATLAS Collaboration, Report No. ATL-PHYS-PUB2010-002.

[16] T. Sjostrand, S. Mrenna, and P.Z. Skands, J. High Energy Phys. 05 (2006) 026.

[17] P. Nason, J. High Energy Phys. 11 (2004) 040; S. Frixione, P. Nason, and C. Oleari, J. High Energy Phys. 11 (2007) 070; S. Frixione, P. Nason, and G. Ridolfi, J. High Energy Phys. 09 (2007) 126.

[18] J. Alwall et al., J. High Energy Phys. 09 (2007) 028.

[19] S. Frixione and B. R. Webber, J. High Energy Phys. 06 (2002) 029; S. Frixione, E. Laenen, and P. Motylinski, J. High Energy Phys. 03 (2006) 092; S. Frixione et al., J. High Energy Phys. 07 (2008) 029.

[20] G. Marchesini et al., Comput. Phys. Commun. 67, 465 (1992); G. Corcella et al., J. High Energy Phys. 01 (2001) 010.

[21] K. Melnikov and F. Petriello, Phys. Rev. D 74, 114017 (2006).

[22] R. Bonciani et al., Nucl. Phys. B529, 424 (1998).

[23] J. Campbell, R. Ellis, and D. Rainwater, Phys. Rev. D 68, 094021 (2003).

[24] P. Langacker, Rev. Mod. Phys. 81, 1199 (2009).

[25] S. Moretti et al., J. High Energy Phys. 04 (2002) 028.

[26] S.-M. Wang et al., Phys. Rev. D 74, 057902 (2006); S. M. Wang et al., Chin. Phys. Lett. 25, 58 (2008).

[27] R. Hamberg, W. L. van Neerven, and T. Matsuura, Nucl. Phys. B359, 343 (1991).

[28] J. Heinrich and L. Lyons, Annu. Rev. Nucl. Part. Sci. 57, 145 (2007).

G. Aad, ${ }^{48}$ B. Abbott, ${ }^{111}$ J. Abdallah, ${ }^{11}$ A. A. Abdelalim, ${ }^{49}$ A. Abdesselam, ${ }^{118}$ O. Abdinov, ${ }^{10}$ B. Abi, ${ }^{112}$ M. Abolins,${ }^{88}$ H. Abramowicz, ${ }^{153}$ H. Abreu, ${ }^{115}$ E. Acerbi, ${ }^{89 a, 89 b}$ B. S. Acharya, ${ }^{164 a, 164 b}$ D. L. Adams, ${ }^{24}$ T. N. Addy, ${ }^{56}$ J. Adelman, ${ }^{175}$ M. Aderholz, ${ }^{99}$ S. Adomeit,${ }^{98}$ P. Adragna,${ }^{75}$ T. Adye, ${ }^{129}$ S. Aefsky, ${ }^{22}$ J. A. Aguilar-Saavedra, ${ }^{124 b, b}$ M. Aharrouche ${ }^{81}$ S. P. Ahlen, ${ }^{21}$ F. Ahles, ${ }^{48}$ A. Ahmad,${ }^{148}$ M. Ahsan,${ }^{40}$ G. Aielli, ${ }^{133 a, 133 b}$ T. Akdogan, ${ }^{18 a}$ T. P. A. Åkesson, ${ }^{79}$ G. Akimoto, ${ }^{155}$ A. V. Akimov, ${ }^{94}$ A. Akiyama, ${ }^{67}$ M. S. Alam, ${ }^{1}$ M. A. Alam, ${ }^{76}$ S. Albrand, ${ }^{55}$ M. Aleksa, ${ }^{29}$ I. N. Aleksandrov,${ }^{65}$ F. Alessandria, ${ }^{89 a}$ C. Alexa, ${ }^{25 a}$ G. Alexander, ${ }^{153}$ G. Alexandre,${ }^{49}$ T. Alexopoulos, ${ }^{9}$ M. Alhroob, ${ }^{20}$ M. Aliev, ${ }^{15}$ G. Alimonti, ${ }^{89 a}$ J. Alison, ${ }^{120}$ M. Aliyev, ${ }^{10}$ P. P. Allport, ${ }^{73}$ S. E. Allwood-Spiers,${ }^{53}$ J. Almond, ${ }^{82}$ A. Aloisio, ${ }^{102 a, 102 b}$ R. Alon, ${ }^{171}$ A. Alonso, ${ }^{79}$ M. G. Alviggi, ${ }^{102 a, 102 b}$ K. Amako,${ }^{66}$ P. Amaral, ${ }^{29}$ C. Amelung, ${ }^{22}$ V. V. Ammosov, ${ }^{128}$ A. Amorim, ${ }^{124 a, c}$ G. Amorós, ${ }^{167}$ N. Amram, ${ }^{153}$ C. Anastopoulos, ${ }^{139}$ T. Andeen, ${ }^{34}$ C. F. Anders, ${ }^{20}$ K. J. Anderson, ${ }^{30}$ A. Andreazza, ${ }^{89 a, 89 b}$ V. Andrei,${ }^{58 a}$ M-L. Andrieux ${ }^{55}$ X. S. Anduaga,${ }^{70}$

A. Angerami, ${ }^{34}$ F. Anghinolfi, ${ }^{29}$ N. Anjos, ${ }^{124 a}$ A. Annovi, ${ }^{47}$ A. Antonaki,${ }^{8}$ M. Antonelli, ${ }^{47}$ S. Antonelli, ${ }^{19 a, 19 b}$ A. Antonov, ${ }^{96}$ J. Antos, ${ }^{144 \mathrm{~b}}$ F. Anulli, ${ }^{132 \mathrm{a}} \mathrm{S}$. Aoun, ${ }^{83} \mathrm{~L}$. Aperio Bella, ${ }^{4} \mathrm{R}$. Apolle, ${ }^{118}$ G. Arabidze, ${ }^{88}$ I. Aracena, ${ }^{143}$ Y. Arai, ${ }^{66}$ A. T. H. Arce, ${ }^{44}$ J. P. Archambault, ${ }^{28}$ S. Arfaoui, ${ }^{29, d}$ J-F. Arguin, ${ }^{14}$ E. Arik, ${ }^{18 a, a}$ M. Arik, ${ }^{18 a}$ A. J. Armbruster,${ }^{87}$ O. Arnaez, ${ }^{81}$ C. Arnault, ${ }^{115}$ A. Artamonov, ${ }^{95}$ G. Artoni,${ }^{132 a, 132 b}$ D. Arutinov, ${ }^{20}$ S. Asai, ${ }^{155}$ R. Asfandiyarov, ${ }^{172}$ S. Ask, ${ }^{27}$ B. Asman, ${ }^{146 a, 146 b}$ L. Asquith, ${ }^{5}$ K. Assamagan ${ }^{24}$ A. Astbury, ${ }^{169}$ A. Astvatsatourov, ${ }^{52}$ G. Atoian, ${ }^{175}$ B. Aubert, ${ }^{4}$ B. Auerbach, ${ }^{175}$ E. Auge, ${ }^{115}$ K. Augsten, ${ }^{127}$ M. Aurousseau, ${ }^{145 a}$ N. Austin, ${ }^{73}$ R. Avramidou, ${ }^{9}$ D. Axen, ${ }^{168}$ C. Ay, ${ }^{54}$ G. Azuelos, ${ }^{93, e}$ Y. Azuma,${ }^{155}$ M. A. Baak, ${ }^{29}$ G. Baccaglioni,${ }^{89 a}$ 
C. Bacci, ${ }^{134 a, 134 b}$ A. M. Bach, ${ }^{14}$ H. Bachacou, ${ }^{136}$ K. Bachas,${ }^{29}$ G. Bachy, ${ }^{29}$ M. Backes,${ }^{49}$ M. Backhaus,${ }^{20}$

E. Badescu, ${ }^{25 a}$ P. Bagnaia, ${ }^{132 a, 132 b}$ S. Bahinipati, ${ }^{2}$ Y. Bai, ${ }^{32 a}$ D. C. Bailey, ${ }^{158}$ T. Bain, ${ }^{158}$ J. T. Baines, ${ }^{129}$

O. K. Baker, ${ }^{175}$ M. D. Baker, ${ }^{24}$ S. Baker, ${ }^{77}$ F. Baltasar Dos Santos Pedrosa, ${ }^{29}$ E. Banas, ${ }^{38}$ P. Banerjee, ${ }^{93}$ Sw. Banerjee, ${ }^{169}$ D. Banfi, ${ }^{29}$ A. Bangert, ${ }^{137}$ V. Bansal, ${ }^{169}$ H. S. Bansil, ${ }^{17}$ L. Barak, ${ }^{171}$ S. P. Baranov, ${ }^{94}$ A. Barashkou, ${ }^{65}$ A. Barbaro Galtieri, ${ }^{14}$ T. Barber, ${ }^{27}$ E. L. Barberio, ${ }^{86}$ D. Barberis, ${ }^{50,50 b}$ M. Barbero, ${ }^{20}$ D. Y. Bardin ${ }^{65}$ T. Barillari, ${ }^{99}$ M. Barisonzi, ${ }^{174}$ T. Barklow, ${ }^{143}$ N. Barlow, ${ }^{27}$ B. M. Barnett, ${ }^{129}$ R. M. Barnett, ${ }^{14}$ A. Baroncelli, ${ }^{134 a}$ A. J. Barr, ${ }^{18}$

F. Barreiro, ${ }^{80}$ J. Barreiro Guimarães da Costa,${ }^{57}$ P. Barrillon, ${ }^{115}$ R. Bartoldus, ${ }^{143}$ A. E. Barton, ${ }^{71}$ D. Bartsch, ${ }^{20}$

V. Bartsch, ${ }^{149}$ R. L. Bates, ${ }^{53}$ L. Batkova, ${ }^{144 a}$ J. R. Batley, ${ }^{27}$ A. Battaglia, ${ }^{16}$ M. Battistin, ${ }^{29}$ G. Battistoni, ${ }^{89 a}$ F. Bauer, ${ }^{136}$ H. S. Bawa, ${ }^{143, f}$ B. Beare, ${ }^{158}$ T. Beau,${ }^{78}$ P. H. Beauchemin, ${ }^{118}$ R. Beccherle, ${ }^{50 a}$ P. Bechtle, ${ }^{41}$ H. P. Beck, ${ }^{16}$ M. Beckingham, ${ }^{48}$ K. H. Becks, ${ }^{174}$ A. J. Beddall, ${ }^{18 c}$ A. Beddall, ${ }^{18 c}$ S. Bedikian, ${ }^{175}$ V. A. Bednyakov, ${ }^{65}$ C. P. Bee, ${ }^{83}$

M. Begel, ${ }^{24}$ S. Behar Harpaz, ${ }^{152}$ P. K. Behera, ${ }^{63}$ M. Beimforde, ${ }^{99}$ C. Belanger-Champagne, ${ }^{166}$ P. J. Bell, ${ }^{49}$

W. H. Bell, ${ }^{49}$ G. Bella, ${ }^{153}$ L. Bellagamba, ${ }^{19 a}$ F. Bellina, ${ }^{29}$ M. Bellomo, ${ }^{19 a}$ A. Belloni,${ }^{57}$ O. Beloborodova, ${ }^{107}$ K. Belotskiy, ${ }^{96}$ O. Beltramello, ${ }^{29}$ S. Ben Ami, ${ }^{152}$ O. Benary, ${ }^{153}$ D. Benchekroun, ${ }^{135 a}$ C. Benchouk,${ }^{83}$ M. Bendel,${ }^{81}$ B. H. Benedict, ${ }^{163}$ N. Benekos, ${ }^{165}$ Y. Benhammou, ${ }^{153}$ D. P. Benjamin, ${ }^{44}$ M. Benoit, ${ }^{115}$ J. R. Bensinger, ${ }^{22}$ K. Benslama ${ }^{130}$ S. Bentvelsen, ${ }^{105}$ D. Berge, ${ }^{29}$ E. Bergeaas Kuutmann, ${ }^{41}$ N. Berger, ${ }^{4}$ F. Berghaus, ${ }^{169}$ E. Berglund ${ }^{49}$ J. Beringer, ${ }^{14}$ K. Bernardet, ${ }^{83}$ P. Bernat, ${ }^{77}$ R. Bernhard ${ }^{48}$ C. Bernius,${ }^{24}$ T. Berry, ${ }^{76}$ A. Bertin, ${ }^{19 a, 19 b}$ F. Bertinelli, ${ }^{29}$ F. Bertolucci, ${ }^{122 a, 122 b}$ M. I. Besana, ${ }^{89 a, 89 b}$ N. Besson, ${ }^{136}$ S. Bethke, ${ }^{99}$ W. Bhimji, ${ }^{45}$ R. M. Bianchi, ${ }^{29}$ M. Bianco,${ }^{72 a, 72 b}$

O. Biebel,,${ }^{98}$ S. P. Bieniek, ${ }^{77}$ J. Biesiada, ${ }^{14}$ M. Biglietti, ${ }^{134 a, 134 b}$ H. Bilokon, ${ }^{47}$ M. Bindi, ${ }^{19 a, 19 b}$ S. Binet,${ }^{115}$

A. Bingul, ${ }^{18 \mathrm{c}}$ C. Bini, ${ }^{132 \mathrm{a}, 132 \mathrm{~b}}$ C. Biscarat, ${ }^{177}$ U. Bitenc, ${ }^{48}$ K. M. Black, ${ }^{21}$ R. E. Blair, ${ }^{5}$ J.-B. Blanchard, ${ }^{115}$

G. Blanchot, ${ }^{29}$ C. Blocker, ${ }^{22}$ J. Blocki,${ }^{38}$ A. Blondel, ${ }^{49}$ W. Blum,${ }^{81}$ U. Blumenschein, ${ }^{54}$ G. J. Bobbink,${ }^{105}$ V. B. Bobrovnikov, ${ }^{107}$ S. S. Bocchetta,${ }^{79}$ A. Bocci, ${ }^{44}$ C. R. Boddy,${ }^{18}$ M. Boehler, ${ }^{41}$ J. Boek, ${ }^{174}$ N. Boelaert,${ }^{35}$ S. Böser ${ }^{77}$ J. A. Bogaerts, ${ }^{29}$ A. Bogdanchikov, ${ }^{107}$ A. Bogouch, ${ }^{90, a}$ C. Bohm,,${ }^{146 a}$ V. Boisvert, ${ }^{76}$ T. Bold, ${ }^{163, g}$ V. Boldea, ${ }^{25 a}$ M. Bona, ${ }^{75}$ V. G. Bondarenko, ${ }^{96}$ M. Boonekamp, ${ }^{136}$ G. Boorman, ${ }^{76}$ C. N. Booth, ${ }^{139}$ P. Booth, ${ }^{139}$

S. Bordoni, ${ }^{78}$ C. Borer ${ }^{16}$ A. Borisov, ${ }^{128}$ G. Borissov, ${ }^{71}$ I. Borjanovic, ${ }^{12 a}$ S. Borroni, ${ }^{132 a, 132 b}$ K. Bos, ${ }^{105}$

D. Boscherini, ${ }^{19 a}$ M. Bosman, ${ }^{11}$ H. Boterenbrood, ${ }^{105}$ D. Botterill, ${ }^{129}$ J. Bouchami, ${ }^{93}$ J. Boudreau, ${ }^{123}$

E. V. Bouhova-Thacker, ${ }^{71}$ C. Boulahouache, ${ }^{123}$ C. Bourdarios, ${ }^{115}$ N. Bousson,${ }^{83}$ A. Boveia, ${ }^{30}$ J. Boyd ${ }^{29}$

I. R. Boyko, ${ }^{65}$ N. I. Bozhko, ${ }^{128}$ I. Bozovic-Jelisavcic, ${ }^{12 b}$ J. Bracinik, ${ }^{17}$ A. Braem, ${ }^{29}$ P. Branchini, ${ }^{134 a}$

G. W. Brandenburg, ${ }^{57}$ A. Brandt, ${ }^{7}$ G. Brandt, ${ }^{15}$ O. Brandt, ${ }^{54}$ U. Bratzler, ${ }^{156}$ B. Brau, ${ }^{84}$ J. E. Brau, ${ }^{114}$ H. M. Braun, ${ }^{174}$ B. Brelier, ${ }^{158}$ J. Bremer, ${ }^{29}$ R. Brenner, ${ }^{166}$ S. Bressler, ${ }^{152}$ D. Breton, ${ }^{115}$ N. D. Brett, ${ }^{118}$ D. Britton, ${ }^{53}$ F. M. Brochu, ${ }^{27}$

I. Brock,${ }^{20}$ R. Brock,${ }^{88}$ T. J. Brodbeck,${ }^{71}$ E. Brodet,${ }^{153}$ F. Broggi, ${ }^{89 a}$ C. Bromberg,${ }^{88}$ G. Brooijmans,${ }^{34}$

W. K. Brooks, ${ }^{31 \mathrm{~b}}$ G. Brown, ${ }^{82}$ E. Brubaker, ${ }^{30}$ P. A. Bruckman de Renstrom, ${ }^{38}$ D. Bruncko, ${ }^{144 \mathrm{~b}}$ R. Bruneliere, ${ }^{48}$ S. Brunet, ${ }^{61}$ A. Bruni, ${ }^{19 a}$ G. Bruni, ${ }^{19 a}$ M. Bruschi, ${ }^{19 a}$ T. Buanes, ${ }^{13}$ F. Bucci, ${ }^{49}$ J. Buchanan, ${ }^{118}$ N. J. Buchanan, ${ }^{2}$ P. Buchholz, ${ }^{141}$ R. M. Buckingham, ${ }^{118}$ A. G. Buckley, ${ }^{45}$ S. I. Buda, ${ }^{25 a}$ I. A. Budagov, ${ }^{65}$ B. Budick, ${ }^{108}$ V. Büscher, ${ }^{81}$ L. Bugge, ${ }^{117}$ D. Buira-Clark, ${ }^{118}$ E. J. Buis, ${ }^{105}$ O. Bulekov, ${ }^{96}$ M. Bunse, ${ }^{42}$ T. Buran,,${ }^{117}$ H. Burckhart, ${ }^{29}$ S. Burdin, ${ }^{73}$ T. Burgess, ${ }^{13}$ S. Burke, ${ }^{129}$ E. Busato, ${ }^{33}$ P. Bussey, ${ }^{53}$ C. P. Buszello, ${ }^{166}$ F. Butin, ${ }^{29}$ B. Butler,${ }^{143}$ J. M. Butler, ${ }^{21}$ C. M. Buttar, ${ }^{53}$ J. M. Butterworth, ${ }^{77}$ W. Buttinger, ${ }^{27}$ T. Byatt, ${ }^{77}$ S. Cabrera Urbán, ${ }^{167}$ D. Caforio, ${ }^{19 a, 19 b}$ O. Cakir, ${ }^{3 a}$ P. Calafiura, ${ }^{14}$ G. Calderini, ${ }^{78}$ P. Calfayan, ${ }^{98}$ R. Calkins, ${ }^{106}$ L. P. Caloba, ${ }^{23 a}$ R. Caloi, ${ }^{132 a, 132 b}$ D. Calvet, ${ }^{33}$ S. Calvet, ${ }^{33}$ R. Camacho Toro, ${ }^{33}$ A. Camard, ${ }^{78}$ P. Camarri, ${ }^{133 a, 133 b}$ M. Cambiaghi, ${ }^{119 a, 119 b}$ D. Cameron, ${ }^{117}$ J. Cammin, ${ }^{20}$

S. Campana, ${ }^{29}$ M. Campanelli, ${ }^{77}$ V. Canale,${ }^{102 a, 102 b}$ F. Canelli, ${ }^{30}$ A. Canepa,${ }^{159 a}$ J. Cantero, ${ }^{80}$ L. Capasso, ${ }^{102 a, 102 b}$ M. D. M. Capeans Garrido, ${ }^{29}$ I. Caprini, ${ }^{25 a}$ M. Caprini, ${ }^{25 a}$ D. Capriotti, ${ }^{99}$ M. Capua, ${ }^{36 a, 36 b}$ R. Caputo, ${ }^{148}$ C. Caramarcu, ${ }^{25 a}$ R. Cardarelli, ${ }^{133 a}$ T. Carli, ${ }^{29}$ G. Carlino, ${ }^{102 a}$ L. Carminati, ${ }^{89 a, 89 b}$ B. Caron, ${ }^{159 a}$ S. Caron ${ }^{48}$ C. Carpentieri, ${ }^{48}$ G. D. Carrillo Montoya, ${ }^{172}$ A. A. Carter,${ }^{75}$ J. R. Carter, ${ }^{27}$ J. Carvalho, ${ }^{124 a, h}$ D. Casadei,,${ }^{108}$ M. P. Casado, ${ }^{11}$ M. Cascella, ${ }^{122 a, 122 b}$ C. Caso, ${ }^{50 a, 50 b, a}$ A. M. Castaneda Hernandez, ${ }^{172}$ E. Castaneda-Miranda, ${ }^{172}$ V. Castillo Gimenez, ${ }^{167}$ N. F. Castro, ${ }^{124 a}$ G. Cataldi ${ }^{72 a}$ F. Cataneo, ${ }^{29}$ A. Catinaccio, ${ }^{29}$ J. R. Catmore, ${ }^{71}$ A. Cattai, ${ }^{29}$ G. Cattani, ${ }^{133 a, 133 b}$ S. Caughron, ${ }^{88}$ D. Cauz, ${ }^{164 a, 164 c}$ A. Cavallari, ${ }^{132 a, 132 b}$ P. Cavalleri, ${ }^{78}$ D. Cavalli, ${ }^{89 a}$ M. Cavalli-Sforza, ${ }^{11}$ V. Cavasinni, ${ }^{122 a, 122 b}$ A. Cazzato, ${ }^{72 a, 72 b}$ F. Ceradini, ${ }^{134 a, 134 b}$ A. S. Cerqueira, ${ }^{23 a}$ A. Cerri, ${ }^{29}$ L. Cerrito ${ }^{75}$ F. Cerutti, ${ }^{47}$ S. A. Cetin, ${ }^{18 b}$ F. Cevenini, ${ }^{102 a, 102 b}$ A. Chafaq, ${ }^{135 a}$ D. Chakraborty, ${ }^{106}$ K. Chan, ${ }^{2}$ B. Chapleau, ${ }^{85}$ J. D. Chapman, ${ }^{27}$ J. W. Chapman, ${ }^{87}$ E. Chareyre, ${ }^{78}$ D. G. Charlton, ${ }^{17}$ V. Chavda, ${ }^{82}$ S. Cheatham, ${ }^{71}$ S. Chekanov, ${ }^{5}$ S. V. Chekulaev, ${ }^{159 a}$ G. A. Chelkov, ${ }^{65}$ M. A. Chelstowska, ${ }^{104}$ C. Chen, ${ }^{64}$ H. Chen, ${ }^{24}$ L. Chen, ${ }^{2}$ S. Chen, ${ }^{32 \mathrm{c}}$ T. Chen, ${ }^{32 \mathrm{c}}$ X. Chen, ${ }^{172}$ S. Cheng, ${ }^{32 \mathrm{a}}$ A. Cheplakov, ${ }^{65}$ V. F. Chepurnov, ${ }^{65}$ R. Cherkaoui El Moursli, ${ }^{135 \mathrm{e}}$ V. Chernyatin, ${ }^{24}$ E. Cheu, ${ }^{6}$ S. L. Cheung, ${ }^{158}$ L. Chevalier, ${ }^{136}$ G. Chiefari, ${ }^{102 a, 102 b}$ L. Chikovani, ${ }^{51}$ J. T. Childers, ${ }^{58 a}$ 
A. Chilingarov, ${ }^{71}$ G. Chiodini, ${ }^{72 a}$ M. V. Chizhov, ${ }^{65}$ G. Choudalakis, ${ }^{30}$ S. Chouridou, ${ }^{137}$ I. A. Christidi, ${ }^{77}$ A. Christov, ${ }^{48}$ D. Chromek-Burckhart, ${ }^{29}$ M. L. Chu, ${ }^{151}$ J. Chudoba, ${ }^{125}$ G. Ciapetti, ${ }^{132 a, 132 b}$ K. Ciba, ${ }^{37}$ A. K. Ciftci, ${ }^{3 a}$

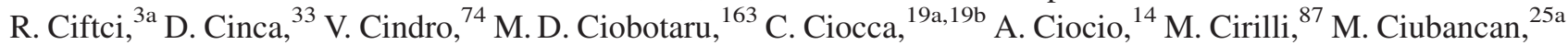
A. Clark, ${ }^{49}$ P. J. Clark, ${ }^{45}$ W. Cleland, ${ }^{123}$ J. C. Clemens, ${ }^{83}$ B. Clement, ${ }^{55}$ C. Clement, ${ }^{146 a, 146 b}$ R. W. Clifft, ${ }^{129}$ Y. Coadou, ${ }^{83}$ M. Cobal, ${ }^{164 a, 164 c}$ A. Coccaro, ${ }^{50 a, 50 b}$ J. Cochran, ${ }^{64}$ P. Coe, ${ }^{118}$ J. G. Cogan, ${ }^{143}$ J. Coggeshall, ${ }^{165}$ E. Cogneras, ${ }^{177}$ C. D. Cojocaru, ${ }^{28}$ J. Colas, ${ }^{4}$ A. P. Colijn, ${ }^{105}$ C. Collard, ${ }^{115}$ N. J. Collins, ${ }^{17}$ C. Collins-Tooth,${ }^{53}$ J. Collot, ${ }^{55}$ G. Colon, ${ }^{84}$ G. Comune, ${ }^{88}$ P. Conde Muiño, ${ }^{124 a}$ E. Coniavitis, ${ }^{118}$ M. C. Conidi, ${ }^{11}$ M. Consonni, ${ }^{104}$ S. Constantinescu, ${ }^{25 a}$ C. Conta, ${ }^{119 a, 119 b}$ F. Conventi, ${ }^{102 a, i}$ J. Cook, ${ }^{29}$ M. Cooke, ${ }^{14}$ B. D. Cooper, ${ }^{77}$

A. M. Cooper-Sarkar, ${ }^{118}$ N. J. Cooper-Smith, ${ }^{76}$ K. Copic, ${ }^{34}$ T. Cornelissen, ${ }^{50 a, 50 b}$ M. Corradi, ${ }^{19 a}$ F. Corriveau, ${ }^{85, j}$ A. Cortes-Gonzalez, ${ }^{165}$ G. Cortiana, ${ }^{99}$ G. Costa,${ }^{89 a}$ M. J. Costa, ${ }^{167}$ D. Costanzo, ${ }^{139}$ T. Costin, ${ }^{30}$ D. Côté, ${ }^{29}$ R. Coura Torres ${ }^{23 a}$ L. Courneyea, ${ }^{169}$ G. Cowan, ${ }^{76}$ C. Cowden,${ }^{27}$ B. E. Cox,${ }^{82}$ K. Cranmer, ${ }^{108}$ F. Crescioli, ${ }^{122 a, 122 b}$ M. Cristinziani, ${ }^{20}$ G. Crosetti, ${ }^{36 a, 36 b}$ R. Crupi,${ }^{72 a, 72 b}$ S. Crépé-Renaudin,${ }^{55}$ C. Cuenca Almenar, ${ }^{175}$ T. Cuhadar Donszelmann, ${ }^{139}$ S. Cuneo,${ }^{50 a, 50 b}$ M. Curatolo, ${ }^{47}$ C. J. Curtis, ${ }^{17}$ P. Cwetanski, ${ }^{61}$ H. Czirr, ${ }^{141}$ Z. Czyczula, ${ }^{117}$ S. D'Auria, ${ }^{53}$ M. D'Onofrio, ${ }^{73}$ A. D'Orazio, ${ }^{132 a, 132 b}$ A. Da Rocha Gesualdi Mello, ${ }^{23 a}$ P. V. M. Da Silva, ${ }^{23 a}$ C. Da Via, ${ }^{82}$ W. Dabrowski, ${ }^{37}$ A. Dahlhoff, ${ }^{48}$ T. Dai, ${ }^{87}$ C. Dallapiccola, ${ }^{84}$ S. J. Dallison, ${ }^{129, a}$ M. Dam, ${ }^{35}$ M. Dameri, ${ }^{50 a, 50 b}$ D. S. Damiani, ${ }^{137}$ H. O. Danielsson, ${ }^{29}$ R. Dankers, ${ }^{105}$ D. Dannheim,,${ }^{99}$ V. Dao, ${ }^{49}$ G. Darbo, ${ }^{50 a}$ G. L. Darlea, ${ }^{25 b}$ C. Daum, ${ }^{105}$ J. P. Dauvergne, ${ }^{29}$ W. Davey, ${ }^{86}$ T. Davidek, ${ }^{126}$ N. Davidson, ${ }^{86}$ R. Davidson, ${ }^{71}$ M. Davies, ${ }^{93}$ A. R. Davison, ${ }^{77}$ E. Dawe, ${ }^{142}$ I. Dawson, ${ }^{139}$ J. W. Dawson, ${ }^{5, a}$ R. K. Daya ${ }^{39}$ K. De, ${ }^{7}$ R. de Asmundis, ${ }^{102 a}$ S. De Castro, ${ }^{19 a, 19 b}$ P. E. De Castro Faria Salgado, ${ }^{24}$ S. De Cecco, ${ }^{78}$ J. de Graat,${ }^{98}$ N. De Groot, ${ }^{104}$ P. de Jong, ${ }^{105}$ C. De La Taille, ${ }^{115}$ H. De la Torre,${ }^{80}$ B. De Lotto, ${ }^{164 a, 164 c}$ L. De Mora, ${ }^{71}$ L. De Nooij, ${ }^{105}$ M. De Oliveira Branco, ${ }^{29}$ D. De Pedis, ${ }^{132 a}$ P. de Saintignon, ${ }^{55}$ A. De Salvo, ${ }^{132 a}$ U. De Sanctis, ${ }^{164 a, 164 c}$ A. De Santo, ${ }^{149}$ J. B. De Vivie De Regie, ${ }^{115}$ S. Dean, ${ }^{77}$ D. V. Dedovich, ${ }^{65}$ J. Degenhardt,${ }^{120}$ M. Dehchar, ${ }^{118}$ M. Deile, ${ }^{98}$ C. Del Papa,${ }^{164 a, 164 c}$ J. Del Peso, ${ }^{80}$ T. Del Prete, ${ }^{122 a, 122 b}$ A. Dell'Acqua, ${ }^{29}$ L. Dell'Asta, ${ }^{89 a, 89 b}$ M. Della Pietra, ${ }^{102 a, i}$ D. della Volpe, ${ }^{102 a, 102 b}$ M. Delmastro, ${ }^{29}$ P. Delpierre,${ }^{83}$ N. Delruelle,${ }^{29}$ P. A. Delsart, ${ }^{55}$ C. Deluca, ${ }^{148}$ S. Demers, ${ }^{175}$ M. Demichev,${ }^{65}$ B. Demirkoz, ${ }^{11}$ J. Deng, ${ }^{163}$ S. P. Denisov, ${ }^{128}$ D. Derendarz,${ }^{38}$ J. E. Derkaoui, ${ }^{135 \mathrm{~d}}$ F. Derue, ${ }^{78}$ P. Dervan,${ }^{73}$ K. Desch, ${ }^{20}$ E. Devetak, ${ }^{148}$ P. O. Deviveiros, ${ }^{158}$ A. Dewhurst, ${ }^{129}$ B. DeWilde, ${ }^{148}$ S. Dhaliwal, ${ }^{158}$ R. Dhullipudi, ${ }^{24, k}$ A. Di Ciaccio, ${ }^{133 a, 133 b}$ L. Di Ciaccio, ${ }^{4}$ A. Di Girolamo, ${ }^{29}$ B. Di Girolamo, ${ }^{29}$ S. Di Luise, ${ }^{134 a, 134 b}$ A. Di Mattia,${ }^{88}$ B. Di Micco, ${ }^{29}$ R. Di Nardo, ${ }^{133 a, 133 b}$ A. Di Simone, ${ }^{133 a, 133 b}$ R. Di Sipio, ${ }^{19 a, 19 b}$ M. A. Diaz, ${ }^{31 \mathrm{a}}$ F. Diblen, ${ }^{18 \mathrm{c}}$ E. B. Diehl,${ }^{87}$ H. Dietl, ${ }^{99}$ J. Dietrich, ${ }^{48}$ T. A. Dietzsch,${ }^{58 a}$ S. Diglio, ${ }^{115}$ K. Dindar Yagci, ${ }^{39}$ J. Dingfelder, ${ }^{20}$ C. Dionisi, ${ }^{132 a, 132 b}$ P. Dita, ${ }^{25 a}$ S. Dita, ${ }^{25 a}$ F. Dittus, ${ }^{29}$ F. Djama, ${ }^{83}$ R. Djilkibaev, ${ }^{108}$

T. Djobava, ${ }^{51}$ M. A. B. do Vale, ${ }^{23 a}$ A. Do Valle Wemans, ${ }^{124 a}$ T. K. O. Doan, ${ }^{4}$ M. Dobbs, ${ }^{85}$ R. Dobinson, ${ }^{29, a}$ D. Dobos,${ }^{42}$ E. Dobson, ${ }^{29}$ M. Dobson, ${ }^{163}$ J. Dodd,${ }^{34}$ O. B. Dogan, ${ }^{18 a, a}$ C. Doglioni, ${ }^{18}$ T. Doherty, ${ }^{53}$ Y. Doi, ${ }^{66, a}$ J. Dolejsi, ${ }^{126}$ I. Dolenc, ${ }^{74}$ Z. Dolezal, ${ }^{126}$ B. A. Dolgoshein, ${ }^{96, a}$ T. Dohmae, ${ }^{155}$ M. Donadelli, ${ }^{23 b}$ M. Donega, ${ }^{120}$ J. Donini, ${ }^{55}$ J. Dopke, ${ }^{29}$ A. Doria, ${ }^{102 a}$ A. Dos Anjos, ${ }^{172}$ M. Dosil, ${ }^{11}$ A. Dotti, ${ }^{122 a, 122 b}$ M. T. Dova, ${ }^{70}$ J. D. Dowell, ${ }^{17}$ A. D. Doxiadis, ${ }^{105}$ A. T. Doyle, ${ }^{53}$ Z. Drasal, ${ }^{126}$ J. Drees, ${ }^{174}$ N. Dressnandt,,${ }^{120}$ H. Drevermann, ${ }^{29}$ C. Driouichi, ${ }^{35}$ M. Dris, ${ }^{9}$ J. G. Drohan, ${ }^{77}$ J. Dubbert, ${ }^{99}$ T. Dubbs, ${ }^{137}$ S. Dube, ${ }^{14}$ E. Duchovni, ${ }^{171}$ G. Duckeck, ${ }^{98}$ A. Dudarev, ${ }^{29}$ F. Dudziak, ${ }^{64}$ M. Dührssen, ${ }^{29}$ I. P. Duerdoth, ${ }^{82}$ L. Duflot,${ }^{15}$ M-A. Dufour, ${ }^{85}$ M. Dunford, ${ }^{29}$ H. Duran Yildiz, ${ }^{3 b}$ R. Duxfield, ${ }^{139}$ M. Dwuznik, ${ }^{37}$ F. Dydak, ${ }^{29}$ D. Dzahini, ${ }^{55}$ M. Düren, ${ }^{52}$ W. L. Ebenstein, ${ }^{44}$ J. Ebke, ${ }^{98}$ S. Eckert, ${ }^{48}$ S. Eckweiler, ${ }^{81}$ K. Edmonds, ${ }^{81}$ C. A. Edwards, ${ }^{76}$ W. Ehrenfeld,${ }^{41}$ T. Ehrich, ${ }^{99}$ T. Eifert, ${ }^{29}$ G. Eigen, ${ }^{13}$ K. Einsweiler,${ }^{14}$ E. Eisenhandler, ${ }^{75}$ T. Ekelof, ${ }^{166}$ M. El Kacimi, ${ }^{4}$ M. Ellert, ${ }^{166}$ S. Elles, ${ }^{4}$ F. Ellinghaus, ${ }^{81}$ K. Ellis, ${ }^{75}$ N. Ellis,${ }^{29}$ J. Elmsheuser ${ }^{98}$ M. Elsing, ${ }^{29}$ R. Ely, ${ }^{14}$ D. Emeliyanov, ${ }^{129}$ R. Engelmann, ${ }^{148}$ A. Engl, ${ }^{98}$ B. Epp ${ }^{62}$ A. Eppig,${ }^{87}$ J. Erdmann, ${ }^{54}$ A. Ereditato, ${ }^{16}$ D. Eriksson, ${ }^{146 a}$ J. Ernst, ${ }^{1}$ M. Ernst,${ }^{24}$ J. Ernwein, ${ }^{136}$ D. Errede, ${ }^{165}$ S. Errede, ${ }^{165}$ E. Ertel,${ }^{81}$ M. Escalier,${ }^{15}$ C. Escobar, ${ }^{167}$ X. Espinal Curull ${ }^{11}$ B. Esposito,${ }^{47}$ F. Etienne, ${ }^{83}$ A. I. Etienvre, ${ }^{136}$

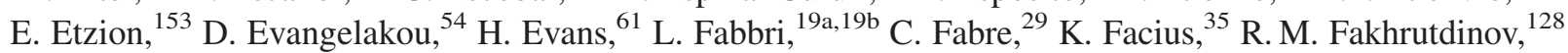
S. Falciano, ${ }^{132 a}$ A. C. Falou, ${ }^{115}$ Y. Fang,${ }^{172}$ M. Fanti,${ }^{89 a, 89 b}$ A. Farbin, ${ }^{7}$ A. Farilla, ${ }^{134 a}$ J. Farley, ${ }^{148}$ T. Farooque, ${ }^{158}$ S. M. Farrington, ${ }^{118}$ P. Farthouat, ${ }^{29}$ D. Fasching, ${ }^{172}$ P. Fassnacht, ${ }^{29}$ D. Fassouliotis,${ }^{8}$ B. Fatholahzadeh, ${ }^{158}$

A. Favareto, ${ }^{89 a, 89 b}$ L. Fayard, ${ }^{115}$ S. Fazio, ${ }^{36 a, 36 b}$ R. Febbraro, ${ }^{33}$ P. Federic, ${ }^{144 a}$ O. L. Fedin, ${ }^{121}$ I. Fedorko, ${ }^{29}$ W. Fedorko, ${ }^{88}$ M. Fehling-Kaschek, ${ }^{48}$ L. Feligioni, ${ }^{83}$ D. Fellmann, ${ }^{5}$ C. U. Felzmann, ${ }^{86}$ C. Feng, ${ }^{32 \mathrm{~d}}$ E. J. Feng ${ }^{30}$ A. B. Fenyuk, ${ }^{128}$ J. Ferencei, ${ }^{144 \mathrm{~b}}$ J. Ferland, ${ }^{93}$ B. Fernandes, ${ }^{124 a, c}$ W. Fernando, ${ }^{109}$ S. Ferrag,${ }^{53}$ J. Ferrando, ${ }^{118}$ V. Ferrara, ${ }^{41}$ A. Ferrari, ${ }^{166}$ P. Ferrari, ${ }^{105}$ R. Ferrari, ${ }^{19 a}$ A. Ferrer, ${ }^{167}$ M. L. Ferrer,${ }^{47}$ D. Ferrere, ${ }^{49}$ C. Ferretti, ${ }^{87}$ A. Ferretto Parodi, ${ }^{50,50 b}$ M. Fiascaris,${ }^{30}$ F. Fiedler, ${ }^{81}$ A. Filipčič,${ }^{74}$ A. Filippas, ${ }^{9}$ F. Filthaut, ${ }^{104}$ M. Fincke-Keeler, ${ }^{169}$ 
M. C. N. Fiolhais, ${ }^{124 a, h}$ L. Fiorini, ${ }^{11}$ A. Firan, ${ }^{39}$ G. Fischer, ${ }^{41}$ P. Fischer, ${ }^{20}$ M. J. Fisher, ${ }^{109}$ S. M. Fisher, ${ }^{129}$

J. Flammer, ${ }^{29}$ M. Flechll, ${ }^{48}$ I. Fleck, ${ }^{141}$ J. Fleckner, ${ }^{81}$ P. Fleischmann, ${ }^{173}$ S. Fleischmann, ${ }^{174}$ T. Flick,,${ }^{174}$ L. R. Flores Castillo, ${ }^{172}$ M. J. Flowerdew, ${ }^{99}$ F. Föhlisch, ${ }^{58 a}$ M. Fokitis, ${ }^{9}$ T. Fonseca Martin, ${ }^{16}$ D. A. Forbush, ${ }^{138}$ A. Formica, ${ }^{136}$ A. Forti, ${ }^{82}$ D. Fortin, ${ }^{159 a}$ J. M. Foster, ${ }^{82}$ D. Fournier, ${ }^{115}$ A. Foussat, ${ }^{29}$ A. J. Fowler, ${ }^{44}$ K. Fowler, ${ }^{137}$ H. Fox, ${ }^{71}$ P. Francavilla, ${ }^{122 a, 122 b}$ S. Franchino, ${ }^{119 a, 119 b}$ D. Francis, ${ }^{29}$ T. Frank, ${ }^{171}$ M. Franklin, ${ }^{57}$ S. Franz, ${ }^{29}$ M. Fraternali, ${ }^{119 a, 119 b}$ S. Fratina, ${ }^{120}$ S. T. French, ${ }^{27}$ R. Froeschl, ${ }^{29}$ D. Froidevaux, ${ }^{29}$ J. A. Frost,${ }^{27}$ C. Fukunaga, ${ }^{156}$ E. Fullana Torregrosa, ${ }^{29}$ J. Fuster, ${ }^{167}$ C. Gabaldon, ${ }^{29}$ O. Gabizon, ${ }^{171}$ T. Gadfort, ${ }^{24}$ S. Gadomski, ${ }^{49}$ G. Gagliardi, ${ }^{50,50 b}$ P. Gagnon, ${ }^{61}$ C. Galea, ${ }^{98}$ E. J. Gallas, ${ }^{118}$ M. V. Gallas, ${ }^{29}$ V. Gallo, ${ }^{16}$ B. J. Gallop, ${ }^{129}$ P. Gallus, ${ }^{125}$ E. Galyaev, ${ }^{40}$ K. K. Gan, ${ }^{109}$ Y. S. Gao, ${ }^{143, f}$ V. A. Gapienko, ${ }^{128}$ A. Gaponenko, ${ }^{14}$ F. Garberson, ${ }^{175}$ M. Garcia-Sciveres,${ }^{14}$ C. García, ${ }^{167}$ J. E. García Navarro, ${ }^{49}$ R. W. Gardner,${ }^{30}$ N. Garelli, ${ }^{29}$ H. Garitaonandia, ${ }^{105}$ V. Garonne, ${ }^{29}$ J. Garvey,${ }^{17}$ C. Gatti ${ }^{47}$ G. Gaudio, ${ }^{19 a}$ O. Gaumer, ${ }^{49}$ B. Gaur, ${ }^{141}$ L. Gauthier, ${ }^{136}$ I. L. Gavrilenko, ${ }^{94}$ C. Gay, ${ }^{168}$ G. Gaycken, ${ }^{20}$ J-C. Gayde, ${ }^{29}$ E. N. Gazis, ${ }^{9}$ P. Ge, ${ }^{32 d}$ C. N. P. Gee,${ }^{129}$ D. A. A. Geerts, ${ }^{105}$ Ch. Geich-Gimbel, ${ }^{20}$ K. Gellerstedt, ${ }^{146 a, 146 b}$

C. Gemme, ${ }^{50 a}$ A. Gemmell, ${ }^{53}$ M. H. Genest,${ }^{98}$ S. Gentile, ${ }^{132 a, 132 b}$ M. George,${ }^{54}$ S. George,${ }^{76}$ P. Gerlach,,${ }^{174}$ A. Gershon, ${ }^{153}$ C. Geweniger, ${ }^{58 a}$ H. Ghazlane, ${ }^{135 b}$ P. Ghez, ${ }^{4}$ N. Ghodbane, ${ }^{33}$ B. Giacobbe, ${ }^{19 a}$ S. Giagu, ${ }^{132 a, 132 b}$ V. Giakoumopoulou, ${ }^{8}$ V. Giangiobbe,${ }^{122 \mathrm{a}, 122 \mathrm{~b}}$ F. Gianotti, ${ }^{29}$ B. Gibbard, ${ }^{24}$ A. Gibson, ${ }^{158}$ S. M. Gibson, ${ }^{29}$ G. F. Gieraltowski, ${ }^{5}$ L. M. Gilbert, ${ }^{118}$ M. Gilchriese, ${ }^{14}$ V. Gilewsky, ${ }^{91}$ D. Gillberg, ${ }^{28}$ A. R. Gillman, ${ }^{129}$ D. M. Gingrich, ${ }^{2, \mathrm{e}}$ J. Ginzburg, ${ }^{153}$ N. Giokaris, ${ }^{8}$ R. Giordano, ${ }^{102 a, 102 b}$ F. M. Giorgi, ${ }^{15}$ P. Giovannini, ${ }^{99}$ P. F. Giraud, ${ }^{136}$ D. Giugni, ${ }^{89 a}$ P. Giusti, ${ }^{19 a}$ B. K. Gjelsten, ${ }^{17}$ L. K. Gladilin, ${ }^{97}$ C. Glasman, ${ }^{80}$ J. Glatzer, ${ }^{48}$ A. Glazov, ${ }^{41}$ K. W. Glitza, ${ }^{174}$ G. L. Glonti, ${ }^{65}$ J. Godfrey, ${ }^{142}$ J. Godlewski, ${ }^{29}$ M. Goebel, ${ }^{41}$ T. Göpfert, ${ }^{43}$ C. Goeringer, ${ }^{81}$ C. Gössling, ${ }^{42}$ T. Göttfert, ${ }^{99}$ S. Goldfarb,${ }^{87}$ D. Goldin, ${ }^{39}$ T. Golling,,${ }^{175}$ S. N. Golovnia, ${ }^{128}$ A. Gomes, ${ }^{124 a, c}$ L. S. Gomez Fajardo, ${ }^{41}$ R. Gonçalo, ${ }^{76}$ J. Goncalves Pinto Firmino Da Costa ${ }^{41}$ L. Gonella, ${ }^{20}$ A. Gonidec,${ }^{29}$ S. Gonzalez, ${ }^{172}$ S. González de la Hoz, ${ }^{167}$ M. L. Gonzalez Silva, ${ }^{26}$ S. Gonzalez-Sevilla, ${ }^{49}$ J. J. Goodson, ${ }^{148}$ L. Goossens, ${ }^{29}$ P. A. Gorbounov, ${ }^{95}$ H. A. Gordon, ${ }^{24}$ I. Gorelov, ${ }^{103}$ G. Gorfine, ${ }^{174}$ B. Gorini, ${ }^{29}$ E. Gorini, ${ }^{72 a, 72 b}$ A. Gorišek, ${ }^{74}$ E. Gornicki, ${ }^{38}$ S. A. Gorokhov, ${ }^{128}$ V. N. Goryachev, ${ }^{128}$ B. Gosdzik, ${ }^{41}$ M. Gosselink, ${ }^{105}$ M. I. Gostkin, ${ }^{65}$ M. Gouanère, ${ }^{4}$ I. Gough Eschrich, ${ }^{163}$ M. Gouighri, ${ }^{135 a}$ D. Goujdami, ${ }^{135 \mathrm{c}}$ M. P. Goulette, ${ }^{49}$ A. G. Goussiou, ${ }^{138}$ C. Goy, ${ }^{4}$ I. Grabowska-Bold, ${ }^{163, \mathrm{~g}}$ V. Grabski, ${ }^{176}$ P. Grafström, ${ }^{29}$ C. Grah, ${ }^{174}$ K-J. Grahn, ${ }^{147}$ F. Grancagnolo, ${ }^{72 a}$ S. Grancagnolo, ${ }^{15}$ V. Grassi, ${ }^{148}$ V. Gratchev, ${ }^{121}$ N. Grau,${ }^{34}$ H. M. Gray, ${ }^{29}$ J. A. Gray, ${ }^{148}$ E. Graziani, ${ }^{134 a}$ O. G. Grebenyuk, ${ }^{121}$ D. Greenfield, ${ }^{129}$ T. Greenshaw, ${ }^{73}$ Z. D. Greenwood, ${ }^{24, k}$ I. M. Gregor, ${ }^{41}$ P. Grenier, ${ }^{143}$ E. Griesmayer, ${ }^{46}$ J. Griffiths, ${ }^{138}$ N. Grigalashvili, ${ }^{65}$ A. A. Grillo, ${ }^{137}$ S. Grinstein, ${ }^{11}$ P. L. Y. Gris, ${ }^{33}$ Y. V. Grishkevich, ${ }^{97}$ J.-F. Grivaz, ${ }^{115}$ J. Grognuz, ${ }^{29}$ M. Groh, ${ }^{99}$ E. Gross, ${ }^{171}$ J. Grosse-Knetter, ${ }^{54}$ J. Groth-Jensen, ${ }^{79}$ M. Gruwe, ${ }^{29}$ K. Grybel, ${ }^{141}$ V. J. Guarino, ${ }^{5}$ D. Guest,${ }^{175}$ C. Guicheney, ${ }^{33}$ A. Guida,${ }^{72 a, 72 b}$ T. Guillemin, ${ }^{4}$ S. Guindon, ${ }^{54}$ H. Guler,${ }^{85,1}$ J. Gunther, ${ }^{125}$ B. Guo, ${ }^{158}$ J. Guo, ${ }^{34}$ A. Gupta,${ }^{30}$ Y. Gusakov ${ }^{65}$ V. N. Gushchin, ${ }^{128}$ A. Gutierrez,${ }^{93}$ P. Gutierrez,${ }^{111}$ N. Guttman, ${ }^{153}$ O. Gutzwiller, ${ }^{172}$ C. Guyot, ${ }^{136}$ C. Gwenlan, ${ }^{118}$ C. B. Gwilliam, ${ }^{73}$ A. Haas, ${ }^{143}$ S. Haas, ${ }^{29}$ C. Haber, ${ }^{14}$ R. Hackenburg, ${ }^{24}$ H. K. Hadavand, ${ }^{39}$ D. R. Hadley, ${ }^{17}$ P. Haefner, ${ }^{99}$ F. Hahn,${ }^{29}$ S. Haider, ${ }^{29}$ Z. Hajduk, ${ }^{38}$ H. Hakobyan, ${ }^{176}$ J. Haller, ${ }^{54}$ K. Hamacher, ${ }^{174}$ P. Hamal, ${ }^{113}$ A. Hamilton, ${ }^{49}$ S. Hamilton, ${ }^{161}$ H. Han, ${ }^{32 a}$ L. Han,${ }^{32 b}$ K. Hanagaki, ${ }^{116}$ M. Hance, ${ }^{120}$ C. Handel, ${ }^{81}$ P. Hanke, ${ }^{58 a}$ C. J. Hansen, ${ }^{166}$ J. R. Hansen, ${ }^{35}$ J. B. Hansen, ${ }^{35}$ J. D. Hansen, ${ }^{35}$ P. H. Hansen, ${ }^{35}$ P. Hansson, ${ }^{143}$ K. Hara, ${ }^{160}$ G. A. Hare, ${ }^{137}$ T. Harenberg, ${ }^{174}$ D. Harper, ${ }^{87}$ R. D. Harrington, ${ }^{21}$ O. M. Harris, ${ }^{138}$ K. Harrison, ${ }^{17}$ J. Hartert, ${ }^{48}$ F. Hartjes, ${ }^{105}$ T. Haruyama, ${ }^{66}$ A. Harvey, ${ }^{56}$ S. Hasegawa, ${ }^{101}$ Y. Hasegawa, ${ }^{140}$ S. Hassani, ${ }^{136}$ M. Hatch, ${ }^{29}$ D. Hauff, ${ }^{99}$ S. Haug, ${ }^{16}$ M. Hauschild, ${ }^{29}$ R. Hauser, ${ }^{88}$ M. Havranek, ${ }^{20}$ B. M. Hawes, ${ }^{118}$ C. M. Hawkes, ${ }^{17}$ R. J. Hawkings, ${ }^{29}$ D. Hawkins, ${ }^{163}$ T. Hayakawa, ${ }^{67}$ D Hayden, ${ }^{76}$ H. S. Hayward, ${ }^{73}$ S. J. Haywood, ${ }^{129}$ E. Hazen, ${ }^{21}$ M. He, ${ }^{32 \mathrm{~d}}$ S. J. Head, ${ }^{17}$ V. Hedberg, ${ }^{79}$ L. Heelan, ${ }^{7}$ S. Heim, ${ }^{88}$ B. Heinemann, ${ }^{14}$ S. Heisterkamp,${ }^{35}$ L. Helary, ${ }^{4}$ M. Heldmann, ${ }^{48}$ M. Heller, ${ }^{115}$ S. Hellman, ${ }^{146 a, 146 b}$ C. Helsens, ${ }^{11}$ R. C. W. Henderson, ${ }^{71}$ M. Henke, ${ }^{58 a}$ A. Henrichs, ${ }^{54}$ A. M. Henriques Correia, ${ }^{29}$ S. Henrot-Versille, ${ }^{15}$ F. Henry-Couannier, ${ }^{83}$ C. Hensel, ${ }^{54}$ T. Hen $\beta,{ }^{174}$ Y. Hernández Jiménez, ${ }^{167}$ R. Herrberg, ${ }^{15}$ A. D. Hershenhorn, ${ }^{152}$ G. Herten, ${ }^{48}$ R. Hertenberger, ${ }^{98}$ L. Hervas,${ }^{29}$ N. P. Hessey, ${ }^{105}$ A. Hidvegi, ${ }^{146 a}$ E. Higón-Rodriguez, ${ }^{167}$ D. Hill,,${ }^{5, a}$ J. C. Hill, ${ }^{27}$ N. Hill,${ }^{5}$ K. H. Hiller, ${ }^{41}$ S. Hillert, ${ }^{20}$ S. J. Hillier ${ }^{17}$ I. Hinchliffe,${ }^{14}$ E. Hines, ${ }^{120}$ M. Hirose, ${ }^{116}$ F. Hirsch, ${ }^{42}$ D. Hirschbuehl, ${ }^{174}$ J. Hobbs, ${ }^{148}$ N. Hod, ${ }^{153}$ M. C. Hodgkinson, ${ }^{139}$ P. Hodgson, ${ }^{139}$ A. Hoecker, ${ }^{29}$ M. R. Hoeferkamp, ${ }^{103}$ J. Hoffman, ${ }^{39}$ D. Hoffmann, ${ }^{83}$ M. Hohlfeld, ${ }^{81}$ M. Holder, ${ }^{141}$ A. Holmes, ${ }^{118}$

S. O. Holmgren, ${ }^{146 a}$ T. Holy, ${ }^{127}$ J. L. Holzbauer, ${ }^{88}$ Y. Homma,${ }^{67}$ L. Hooft van Huysduynen, ${ }^{108}$ T. Horazdovsky, ${ }^{127}$ C. Horn, ${ }^{143}$ S. Horner,${ }^{48}$ K. Horton, ${ }^{118}$ J-Y. Hostachy,${ }^{55}$ S. Hou, ${ }^{151}$ M. A. Houlden ${ }^{73}$ A. Hoummada, ${ }^{135 a}$ J. Howarth, ${ }^{82}$ D. F. Howell, ${ }^{118}$ I. Hristova, ${ }^{41}$ J. Hrivnac, ${ }^{115}$ I. Hruska, ${ }^{125}$ T. Hryn'ova, ${ }^{4}$ P. J. Hsu, ${ }^{175}$ S.-C. Hsu, ${ }^{14}$ 
G. S. Huang, ${ }^{111}$ Z. Hubacek, ${ }^{127}$ F. Hubaut,${ }^{83}$ F. Huegging, ${ }^{20}$ T. B. Huffman, ${ }^{118}$ E. W. Hughes,${ }^{34}$ G. Hughes, ${ }^{71}$ R. E. Hughes-Jones, ${ }^{82}$ M. Huhtinen, ${ }^{29}$ P. Hurst,${ }^{57}$ M. Hurwitz, ${ }^{14}$ U. Husemann,${ }^{41}$ N. Huseynov, ${ }^{65, m}$ J. Huston, ${ }^{88}$ J. Huth, ${ }^{57}$ G. Iacobucci, ${ }^{102 a}$ G. Iakovidis, ${ }^{9}$ M. Ibbotson, ${ }^{82}$ I. Ibragimov, ${ }^{141}$ R. Ichimiya ${ }^{67}$ L. Iconomidou-Fayard, ${ }^{115}$ J. Idarraga, ${ }^{15} \mathrm{M}$. Idzik, ${ }^{37}$ P. Iengo, ${ }^{102 a, 102 b}$ O. Igonkina, ${ }^{105}$ Y. Ikegami, ${ }^{66} \mathrm{M}$. Ikeno, ${ }^{66}$ Y. Ilchenko,${ }^{39} \mathrm{D}$. Iliadis, ${ }^{154}$ D. Imbault, ${ }^{78} \mathrm{M}$. Imhaeuser, ${ }^{174} \mathrm{M}$. Imori, ${ }^{155} \mathrm{~T}$. Ince, ${ }^{20} \mathrm{~J}$. Inigo-Golfin, ${ }^{29} \mathrm{P}$. Ioannou, ${ }^{8} \mathrm{M}$. Iodice, ${ }^{134 \mathrm{a}} \mathrm{G}$. Ionescu, ${ }^{4}$ A. Irles Quiles, ${ }^{167} \mathrm{~K}$. Ishii, ${ }^{66}$ A. Ishikawa, ${ }^{67} \mathrm{M}$. Ishino, ${ }^{66} \mathrm{R}$. Ishmukhametov, ${ }^{39} \mathrm{C}$. Issever, ${ }^{118} \mathrm{~S}$. Istin, ${ }^{18 a} \mathrm{Y}$. Itoh, ${ }^{101}$ A. V. Ivashin, ${ }^{128}$ W. Iwanski, ${ }^{38}$ H. Iwasaki, ${ }^{66}$ J. M. Izen, ${ }^{40}$ V. Izzo, ${ }^{102 a}$ B. Jackson, ${ }^{120}$ J. N. Jackson, ${ }^{73}$ P. Jackson, ${ }^{143}$ M. R. Jaekel, ${ }^{29}$ V. Jain,${ }^{61}$ K. Jakobs,${ }^{48}$ S. Jakobsen, ${ }^{35}$ J. Jakubek, ${ }^{127}$ D. K. Jana, ${ }^{111}$ E. Jankowski, ${ }^{158}$ E. Jansen, ${ }^{77}$ A. Jantsch, ${ }^{99}$ M. Janus, ${ }^{20}$ G. Jarlskog ${ }^{79}$ L. Jeanty,${ }^{57}$ K. Jelen, ${ }^{37}$ I. Jen-La Plante,${ }^{30}$ P. Jenni, ${ }^{29}$ A. Jeremie, ${ }^{4}$ P. Jež,${ }^{35}$ S. Jézéquel, ${ }^{4}$ M. K. Jha, ${ }^{19 a} \mathrm{H}$. Ji, ${ }^{172}$ W. Ji, ${ }^{81}$ J. Jia, ${ }^{148}$ Y. Jiang, ${ }^{32 b}$ M. Jimenez Belenguer, ${ }^{41}$ G. Jin, ${ }^{32 b}$ S. Jin, ${ }^{32 a}$ O. Jinnouchi, ${ }^{157}$ M. D. Joergensen, ${ }^{35}$ D. Joffe, ${ }^{39}$ L. G. Johansen, ${ }^{13}$ M. Johansen, ${ }^{146 a, 146 b}$ K. E. Johansson, ${ }^{146 a}$ P. Johansson, ${ }^{139}$ S. Johnert, ${ }^{41}$ K. A. Johns, ${ }^{6}$ K. Jon-And, ${ }^{146 a, 146 b}$ G. Jones, ${ }^{82}$ R. W. L. Jones, ${ }^{71}$ T. W. Jones, ${ }^{77}$ T. J. Jones, ${ }^{73}$ O. Jonsson, ${ }^{29}$ C. Joram,${ }^{29}$ P. M. Jorge,,${ }^{124 a, c}$ J. Joseph,${ }^{14}$ X. Ju, ${ }^{130}$ V. Juranek, ${ }^{125}$ P. Jussel,,${ }^{62}$ V. V. Kabachenko, ${ }^{128}$ S. Kabana, ${ }^{16}$ M. Kaci, ${ }^{167}$ A. Kaczmarska, ${ }^{38}$ P. Kadlecik, ${ }^{35}$ M. Kado, ${ }^{115}$ H. Kagan,,${ }^{109}$ M. Kagan ${ }^{57}$ S. Kaiser, ${ }^{99}$ E. Kajomovitz, ${ }^{152}$ S. Kalinin,,${ }^{174}$ L. V. Kalinovskaya, ${ }^{65}$ S. Kama, ${ }^{39}$ N. Kanaya, ${ }^{155}$ M. Kaneda, ${ }^{155}$ T. Kanno, ${ }^{157}$ V. A. Kantserov, ${ }^{96}$ J. Kanzaki, ${ }^{66}$ B. Kaplan, ${ }^{175}$ A. Kapliy, ${ }^{30}$ J. Kaplon, ${ }^{29}$ D. Kar, ${ }^{43}$ M. Karagoz, ${ }^{118}$ M. Karnevskiy, ${ }^{41}$ K. Karr, ${ }^{5}$ V. Kartvelishvili, ${ }^{71}$ A. N. Karyukhin, ${ }^{128}$ L. Kashif, ${ }^{172}$ A. Kasmi, ${ }^{39}$ R. D. Kass, ${ }^{109}$ A. Kastanas, ${ }^{13}$ M. Kataoka, ${ }^{4}$ Y. Kataoka, ${ }^{155}$ E. Katsoufis,${ }^{9}$ J. Katzy, ${ }^{41}$ V. Kaushik, ${ }^{6}$ K. Kawagoe, ${ }^{67}$ T. Kawamoto, ${ }^{155}$ G. Kawamura, ${ }^{81}$ M. S. Kayl, ${ }^{105}$ V. A. Kazanin,,${ }^{107}$ M. Y. Kazarinov, ${ }^{65}$ S. I. Kazi, ${ }^{86}$ J. R. Keates, ${ }^{82}$ R. Keeler, ${ }^{169}$ R. Kehoe,${ }^{39}$ M. Keil,${ }^{54}$ G. D. Kekelidze, ${ }^{65}$ M. Kelly, ${ }^{82}$ J. Kennedy, ${ }^{98}$ C. J. Kenney, ${ }^{143}$ M. Kenyon, ${ }^{53}$ O. Kepka, ${ }^{125}$ N. Kerschen, ${ }^{29}$ B.P. Kerševan, ${ }^{74}$ S. Kersten, ${ }^{174}$ K. Kessoku, ${ }^{155}$ C. Ketterer,${ }^{48}$ M. Khakzad,${ }^{28}$ F. Khalil-zada, ${ }^{10}$ H. Khandanyan, ${ }^{165}$ A. Khanov, ${ }^{112}$ D. Kharchenko, ${ }^{65}$ A. Khodinov, ${ }^{148}$ A. G. Kholodenko, ${ }^{128}$ A. Khomich, ${ }^{58 a}$ T. J. Khoo, ${ }^{27}$ G. Khoriauli, ${ }^{20}$ N. Khovanskiy, ${ }^{65}$ V. Khovanskiy, ${ }^{95}$ E. Khramov, ${ }^{65}$ J. Khubua, ${ }^{51}$ G. Kilvington, ${ }^{76}$ H. Kim, ${ }^{7}$ M. S. Kim,${ }^{2}$ P. C. Kim, ${ }^{143}$ S. H. Kim, ${ }^{160}$ N. Kimura, ${ }^{170}$ O. Kind,${ }^{15}$ B. T. King, ${ }^{73}$ M. King,${ }^{67}$ R. S. B. King, ${ }^{118}$ J. Kirk, ${ }^{129}$ G. P. Kirsch, ${ }^{18}$ L. E. Kirsch, ${ }^{22}$ A. E. Kiryunin, ${ }^{99}$ D. Kisielewska, ${ }^{37}$ T. Kittelmann, ${ }^{123}$ A. M. Kiver, ${ }^{128}$ H. Kiyamura, ${ }^{67}$ E. Kladiva, ${ }^{14 \mathrm{~b}}$ J. Klaiber-Lodewigs, ${ }^{42}$ M. Klein, ${ }^{73}$ U. Klein, ${ }^{73}$ K. Kleinknecht,${ }^{81}$ M. Klemetti, ${ }^{85}$ A. Klier, ${ }^{171}$ A. Klimentov, ${ }^{24}$ R. Klingenberg, ${ }^{42}$ E. B. Klinkby, ${ }^{35}$ T. Klioutchnikova, ${ }^{29}$ P. F. Klok, ${ }^{104}$ S. Klous, ${ }^{105}$ E.-E. Kluge, ${ }^{58 a}$ T. Kluge, ${ }^{73}$ P. Kluit, ${ }^{105}$ S. Kluth, ${ }^{99}$ E. Kneringer, ${ }^{62}$ J. Knobloch, ${ }^{29}$ E. B. F. G. Knoops ${ }^{83}$ A. Knue,${ }^{54}$ B. R. Ko, ${ }^{44}$ T. Kobayashi, ${ }^{155}$ M. Kobel, ${ }^{43}$ B. Koblitz, ${ }^{29}$ M. Kocian, ${ }^{143}$ A. Kocnar, ${ }^{113}$ P. Kodys, ${ }^{126}$ K. Köneke ${ }^{29}$ A. C. König, ${ }^{104}$ S. Koenig, ${ }^{81}$ L. Köpke, ${ }^{81}$ F. Koetsveld, ${ }^{104}$ P. Koevesarki, ${ }^{20}$ T. Koffas, ${ }^{29}$ E. Koffeman, ${ }^{105}$ F. Kohn, ${ }^{54}$ Z. Kohout, ${ }^{127}$ T. Kohriki, ${ }^{66}$ T. Koi, ${ }^{143}$ T. Kokott, ${ }^{20}$ G. M. Kolachev, ${ }^{107}$ H. Kolanoski, ${ }^{15}$ V. Kolesnikov, ${ }^{65}$ I. Koletsou, ${ }^{89 a}$ J. Koll, ${ }^{88}$ D. Kollar, ${ }^{29}$ M. Kollefrath, ${ }^{48}$ S. D. Kolya, ${ }^{82}$ A. A. Komar, ${ }^{94}$ J. R. Komaragiri, ${ }^{142}$ T. Kondo, ${ }^{66}$ T. Kono, ${ }^{41, n}$ A. I. Kononov, ${ }^{48}$ R. Konoplich, ${ }^{108, o}$ N. Konstantinidis, ${ }^{77}$ A. Kootz, ${ }^{174}$ S. Koperny, ${ }^{37}$ S. V. Kopikov, ${ }^{128}$ K. Korcyl,${ }^{38}$ K. Kordas, ${ }^{154}$ V. Koreshev, ${ }^{128}$ A. Korn, ${ }^{14}$ A. Korol, ${ }^{107}$ I. Korolkov, ${ }^{11}$ E. V. Korolkova, ${ }^{139}$ V. A. Korotkov, ${ }^{128}$ O. Kortner, ${ }^{99}$ S. Kortner, ${ }^{99}$ V. V. Kostyukhin, ${ }^{20}$ M. J. Kotamäki, ${ }^{29}$ S. Kotov, ${ }^{99}$ V. M. Kotov, ${ }^{65}$ C. Kourkoumelis, ${ }^{8}$ V. Kouskoura, ${ }^{154}$ A. Koutsman, ${ }^{105}$ R. Kowalewski, ${ }^{169}$ H. Kowalski, ${ }^{41}$ T.Z. Kowalski, ${ }^{37}$ W. Kozanecki, ${ }^{136}$ A. S. Kozhin, ${ }^{128}$ V. Kral, ${ }^{127}$ V. A. Kramarenko, ${ }^{97}$ G. Kramberger, ${ }^{74}$ O. Krasel, ${ }^{42}$ M. W. Krasny, ${ }^{78}$ A. Krasznahorkay, ${ }^{108}$ J. Kraus, ${ }^{88}$ A. Kreisel,,${ }^{153}$ F. Krejci, ${ }^{127}$ J. Kretzschmar, ${ }^{73}$ N. Krieger, ${ }^{54}$ P. Krieger,,${ }^{158}$ K. Kroeninger, ${ }^{54}$ H. Kroha, ${ }^{99}$ J. Kroll, ${ }^{120}$ J. Kroseberg, ${ }^{20}$ J. Krstic, ${ }^{12 a}$ U. Kruchonak, ${ }^{65}$ H. Krüger, ${ }^{20}$ Z. V. Krumshteyn, ${ }^{65}$ A. Kruth, ${ }^{20}$ T. Kubota,${ }^{155}$ S. Kuehn, ${ }^{48}$ A. Kugel,${ }^{58 c}$ T. Kuhl,${ }^{174}$ D. Kuhn, ${ }^{62}$ V. Kukhtin, ${ }^{65}$ Y. Kulchitsky, ${ }^{90}$ S. Kuleshov, ${ }^{31 b}$ C. Kummer, ${ }^{98}$ M. Kuna, ${ }^{78}$ N. Kundu, ${ }^{118}$ J. Kunkle, ${ }^{120}$ A. Kupco, ${ }^{125}$ H. Kurashige, ${ }^{67}$ M. Kurata, ${ }^{160}$ Y. A. Kurochkin, ${ }^{90}$ V. Kus, ${ }^{125}$ W. Kuykendall, ${ }^{138}$ M. Kuze, ${ }^{157}$ P. Kuzhir, ${ }^{91}$ O. Kvasnicka, ${ }^{125}$ J. Kvita, ${ }^{29}$ R. Kwee, ${ }^{15}$ A. La Rosa ${ }^{29}$ L. La Rotonda, ${ }^{36 a, 36 b}$ L. Labarga ${ }^{80}$ J. Labbe, ${ }^{4}$ S. Lablak, ${ }^{135 a}$ C. Lacasta, ${ }^{167}$ F. Lacava, ${ }^{132 \mathrm{a}, 132 \mathrm{~b}}$ H. Lacker, ${ }^{15}$ D. Lacour, ${ }^{78}$ V. R. Lacuesta, ${ }^{167}$ E. Ladygin, ${ }^{65}$ R. Lafaye, ${ }^{4}$ B. Laforge,${ }^{78}$ T. Lagouri, ${ }^{80}$ S. Lai, ${ }^{48}$ E. Laisne ${ }^{55}$ M. Lamanna, ${ }^{29}$ C. L. Lampen, ${ }^{6}$ W. Lampl,${ }^{6}$ E. Lancon, ${ }^{136}$ U. Landgraf, ${ }^{48}$ M. P. J. Landon, ${ }^{75}$ H. Landsman, ${ }^{152}$ J. L. Lane, ${ }^{82}$ C. Lange,${ }^{41}$ A. J. Lankford, ${ }^{163}$ F. Lanni, ${ }^{24}$ K. Lantzsch, ${ }^{29}$ V. V. Lapin, ${ }^{128, a}$ S. Laplace ${ }^{78}$ C. Lapoire, ${ }^{20}$ J. F. Laporte, ${ }^{136}$ T. Lari, ${ }^{89 a}$ A. V. Larionov, ${ }^{128}$ A. Larner, ${ }^{118}$ C. Lasseur, ${ }^{29}$ M. Lassnig, ${ }^{29}$ W. Lau, ${ }^{118}$ P. Laurelli, ${ }^{47}$ A. Lavorato, ${ }^{118}$ W. Lavrijsen, ${ }^{14}$ P. Laycock, ${ }^{73}$ A. B. Lazarev ${ }^{65}$ A. Lazzaro, ${ }^{89 a, 89 b}$ O. Le Dortz, ${ }^{78}$ E. Le Guirriec, ${ }^{83}$ C. Le Maner, ${ }^{158}$ E. Le Menedeu, ${ }^{136}$ A. Lebedev, ${ }^{64}$ C. Lebel,${ }^{93}$ T. LeCompte, ${ }^{5}$ F. Ledroit-Guillon, ${ }^{55}$ H. Lee, ${ }^{105}$ J. S. H. Lee, ${ }^{150}$ S. C. Lee,${ }^{151}$ L. Lee, ${ }^{175}$ M. Lefebvre, ${ }^{169}$ M. Legendre, ${ }^{136}$ A. Leger ${ }^{49}$ B. C. LeGeyt,${ }^{120}$ F. Legger, ${ }^{98}$ C. Leggett, ${ }^{14}$ M. Lehmacher, ${ }^{20}$ G. Lehmann Miotto, ${ }^{29}$ X. Lei, ${ }^{6}$ M. A. L. Leite, ${ }^{23 b}$ R. Leitner, ${ }^{126}$ 
D. Lellouch, ${ }^{171}$ J. Lellouch, ${ }^{78}$ M. Leltchouk,${ }^{34}$ V. Lendermann, ${ }^{58 a}$ K. J. C. Leney, ${ }^{145 b}$ T. Lenz, ${ }^{174}$ G. Lenzen, ${ }^{174}$ B. Lenzi, ${ }^{136}$ K. Leonhardt, ${ }^{43}$ S. Leontsinis, ${ }^{9}$ C. Leroy, ${ }^{93}$ J-R. Lessard, ${ }^{169}$ J. Lesser, ${ }^{146 a}$ C. G. Lester, ${ }^{27}$

A. Leung Fook Cheong, ${ }^{172}$ J. Levêque,${ }^{4}$ D. Levin, ${ }^{87}$ L. J. Levinson,,${ }^{171}$ M. S. Levitski, ${ }^{128}$ M. Lewandowska, ${ }^{21}$ G. H. Lewis, ${ }^{108}$ M. Leyton, ${ }^{15}$ B. Li,${ }^{83}$ H. Li, ${ }^{172}$ S. Li,${ }^{32 b}$ X. Li ${ }^{87}$ Z. Liang, ${ }^{39}$ Z. Liang, ${ }^{18, p}$ B. Liberti, ${ }^{133 a}$ P. Lichard, ${ }^{29}$ M. Lichtnecker ${ }^{98}$ K. Lie, ${ }^{165}$ W. Liebig, ${ }^{13}$ R. Lifshitz, ${ }^{152}$ J. N. Lilley, ${ }^{17}$ C. Limbach, ${ }^{20}$ A. Limosani, ${ }^{86}$ M. Limper, ${ }^{63}$ S. C. Lin, ${ }^{151, \mathrm{q}}$ F. Linde, ${ }^{105}$ J. T. Linnemann,${ }^{88}$ E. Lipeles,${ }^{120}$ L. Lipinsky, ${ }^{125}$ A. Lipniacka, ${ }^{13}$ T. M. Liss,${ }^{165}$ D. Lissauer, ${ }^{24}$ A. Lister, ${ }^{49}$ A. M. Litke, ${ }^{137}$ C. Liu, ${ }^{28}$ D. Liu, ${ }^{151, r}$ H. Liu, ${ }^{87}$ J. B. Liu, ${ }^{87}$ M. Liu, ${ }^{32 b}$ S. Liu, ${ }^{2}$ Y. Liu, ${ }^{32 b}$ M. Livan, ${ }^{119 a, 119 b}$ S. S. A. Livermore, ${ }^{118}$ A. Lleres, ${ }^{55}$ S. L. Lloyd,${ }^{75}$ E. Lobodzinska, ${ }^{41}$ P. Loch, ${ }^{6}$ W. S. Lockman, ${ }^{137}$ S. Lockwitz, ${ }^{175}$ T. Loddenkoetter, ${ }^{20}$ F. K. Loebinger, ${ }^{82}$ A. Loginov, ${ }^{175}$ C. W. Loh, ${ }^{168}$ T. Lohse, ${ }^{15}$ K. Lohwasser ${ }^{48}$ M. Lokajicek, ${ }^{125}$ J. Loken, ${ }^{118}$ V. P. Lombardo, ${ }^{89 a}$ R. E. Long, ${ }^{71}$ L. Lopes, ${ }^{124 a, c}$ D. Lopez Mateos, ${ }^{34, s}$ M. Losada, ${ }^{162}$ P. Loscutoff, ${ }^{14}$ F. Lo Sterzo, ${ }^{132 a, 132 b}$ M. J. Losty, ${ }^{159 a}$ X. Lou,${ }^{40}$ A. Lounis, ${ }^{115}$ K. F. Loureiro, ${ }^{162}$ J. Love, ${ }^{21}$ P. A. Love ${ }^{71}$ A. J. Lowe,${ }^{143, f}$ F. Lu, ${ }^{32 a}$ L. Lu, ${ }^{39}$ H. J. Lubatti, ${ }^{138}$ C. Luci, ${ }^{132 a, 132 b}$ A. Lucotte, ${ }^{55}$ A. Ludwig, ${ }^{43}$ D. Ludwig, ${ }^{41}$ I. Ludwig, ${ }^{48}$ J. Ludwig, ${ }^{48}$ F. Luehring, ${ }^{61}$ G. Luijckx, ${ }^{105}$ D. Lumb, ${ }^{48}$ L. Luminari, ${ }^{132 a}$ E. Lund, ${ }^{117}$ B. Lund-Jensen, ${ }^{147}$ B. Lundberg, ${ }^{79}$ J. Lundberg, ${ }^{146 a, 146 b}$ J. Lundquist, ${ }^{35}$ M. Lungwitz, ${ }^{81}$ A. Lupi, ${ }^{122 a, 122 b}$ G. Lutz, ${ }^{99}$ D. Lynn, ${ }^{24}$ J. Lys,${ }^{14}$ E. Lytken, ${ }^{79}$ H. Ma,${ }^{24}$ L. L. Ma,${ }^{172}$ J. A. Macana Goia, ${ }^{93}$ G. Maccarrone, ${ }^{47}$ A. Macchiolo, ${ }^{99}$ B. Maček, ${ }^{74}$ J. Machado Miguens, ${ }^{124 a}$ D. Macina, ${ }^{49}$ R. Mackeprang,${ }^{35}$ R. J. Madaras, ${ }^{14}$ W. F. Mader, ${ }^{43}$ R. Maenner ${ }^{58 c}$ T. Maeno, ${ }^{24}$ P. Mättig, ${ }^{174}$ S. Mättig, ${ }^{41}$ P. J. Magalhaes Martins, ${ }^{124 a, h}$ L. Magnoni, ${ }^{29}$ E. Magradze, ${ }^{51}$ Y. Mahalalel, ${ }^{153}$ K. Mahboubi, ${ }^{48}$ G. Mahout, ${ }^{17}$ C. Maiani, ${ }^{132 a, 132 b}$ C. Maidantchik, ${ }^{23 a}$ A. Maio, ${ }^{124 a, c}$ S. Majewski, ${ }^{24}$ Y. Makida, ${ }^{66}$ N. Makovec, ${ }^{115}$ P. Mal,${ }^{6}$ Pa. Malecki,${ }^{38}$ P. Malecki,${ }^{38}$ V. P. Maleev, ${ }^{121}$ F. Malek, ${ }^{55}$ U. Mallik, ${ }^{63}$ D. Malon, ${ }^{5}$ S. Maltezos, ${ }^{9}$ V. Malyshev, ${ }^{107}$ S. Malyukov, ${ }^{65}$ R. Mameghani,${ }^{98}$ J. Mamuzic, ${ }^{12 b}$ A. Manabe,${ }^{66}$ L. Mandelli, ${ }^{89 a}$ I. Mandić, ${ }^{74}$ R. Mandrysch, ${ }^{15}$ J. Maneira, ${ }^{124 a}$ P. S. Mangeard, ${ }^{88}$ I. D. Manjavidze, ${ }^{65}$ A. Mann, ${ }^{54}$ P. M. Manning, ${ }^{137}$ A. Manousakis-Katsikakis, ${ }^{8}$ B. Mansoulie, ${ }^{136}$ A. Manz,${ }^{99}$ A. Mapelli, ${ }^{29}$ L. Mapelli, ${ }^{29}$ L. March, ${ }^{80}$ J. F. Marchand, ${ }^{29}$ F. Marchese, ${ }^{133 a, 133 b}$ G. Marchiori, ${ }^{78}$ M. Marcisovsky, ${ }^{125}$ A. Marin, ${ }^{21, a}$ C. P. Marino, ${ }^{61}$ F. Marroquim, ${ }^{23 a}$ R. Marshall, ${ }^{82}$ Z. Marshall, ${ }^{34, \mathrm{~s}}$ F. K. Martens, ${ }^{158}$ S. Marti-Garcia, ${ }^{167}$ A. J. Martin, ${ }^{175}$ B. Martin, ${ }^{29}$ B. Martin, ${ }^{88}$ F. F. Martin, ${ }^{120}$ J. P. Martin, ${ }^{93}$ Ph. Martin, ${ }^{55}$ T. A. Martin, ${ }^{17}$ B. Martin dit Latour, ${ }^{49}$ M. Martinez, ${ }^{11}$ V. Martinez Outschoorn, ${ }^{57}$ A. C. Martyniuk, ${ }^{82}$ M. Marx, ${ }^{82}$ F. Marzano, ${ }^{132 a}$ A. Marzin, ${ }^{111}$ L. Masetti, ${ }^{81}$

T. Mashimo, ${ }^{155}$ R. Mashinistov, ${ }^{94}$ J. Masik, ${ }^{82}$ A. L. Maslennikov, ${ }^{107}$ M. Maß, ${ }^{42}$ I. Massa, ${ }^{19 a, 19 b}$ G. Massaro, ${ }^{105}$ N. Massol, ${ }^{4}$ A. Mastroberardino, ${ }^{36 a, 36 b}$ T. Masubuchi, ${ }^{155}$ M. Mathes,${ }^{20}$ P. Matricon, ${ }^{115}$ H. Matsumoto, ${ }^{155}$ H. Matsunaga, ${ }^{155}$ T. Matsushita ${ }^{67}$ C. Mattravers,,${ }^{118, t}$ J. M. Maugain, ${ }^{29}$ S. J. Maxfield, ${ }^{73}$ D. A. Maximov, ${ }^{107}$ E. N. May ${ }^{5}$ A. Mayne, ${ }^{139}$ R. Mazini, ${ }^{151}$ M. Mazur, ${ }^{20}$ M. Mazzanti, ${ }^{89 a}$ E. Mazzoni, ${ }^{122 a, 122 b}$ S. P. Mc Kee, ${ }^{87}$ A. McCarn, ${ }^{165}$ R. L. McCarthy, ${ }^{148}$ T. G. McCarthy, ${ }^{28}$ N. A. McCubbin, ${ }^{129}$ K. W. McFarlane, ${ }^{56}$ J. A. Mcfayden, ${ }^{139}$ H. McGlone, ${ }^{53}$ G. Mchedlidze, ${ }^{51}$ R. A. McLaren, ${ }^{29}$ T. Mclaughlan, ${ }^{17}$ S. J. McMahon, ${ }^{129}$ R. A. McPherson, ${ }^{169, j}$ A. Meade,${ }^{84}$ J. Mechnich, ${ }^{105}$ M. Mechtel,,${ }^{174}$ M. Medinnis, ${ }^{41}$ R. Meera-Lebbai, ${ }^{111}$ T. Meguro, ${ }^{116}$ R. Mehdiyev, ${ }^{93}$ S. Mehlhase, ${ }^{35}$ A. Mehta, ${ }^{73}$ K. Meier,${ }^{58 a}$ J. Meinhardt,${ }^{48}$ B. Meirose,${ }^{79}$ C. Melachrinos,${ }^{30}$ B. R. Mellado Garcia, ${ }^{172}$ L. Mendoza Navas, ${ }^{162}$ Z. Meng, ${ }^{151, \mathrm{r}}$ A. Mengarelli, ${ }^{19 a, 19 b}$ S. Menke, ${ }^{99}$ C. Menot,${ }^{29}$ E. Meoni, ${ }^{11}$ K. M. Mercurio, ${ }^{57}$ P. Mermod, ${ }^{118}$ L. Merola, ${ }^{102 a, 102 b}$ C. Meroni, ${ }^{89 a}$ F. S. Merritt,${ }^{30}$ A. Messina, ${ }^{29}$ J. Metcalfe, ${ }^{103}$ A. S. Mete, ${ }^{64}$ S. Meuser ${ }^{20}$ C. Meyer, ${ }^{81}$ J-P. Meyer, ${ }^{136}$ J. Meyer, ${ }^{173}$ J. Meyer, ${ }^{54}$ T. C. Meyer, ${ }^{29}$ W. T. Meyer, ${ }^{64}$ J. Miao, ${ }^{32 d}$ S. Michal,${ }^{29}$ L. Micu, ${ }^{25 a}$ R. P. Middleton, ${ }^{129}$ P. Miele, ${ }^{29}$ S. Migas, ${ }^{73}$ L. Mijović,${ }^{41}$ G. Mikenberg, ${ }^{171}$

M. Mikestikova, ${ }^{125}$ B. Mikulec, ${ }^{49}$ M. Mikuž,${ }^{74}$ D. W. Miller, ${ }^{143}$ R. J. Miller,${ }^{88}$ W. J. Mills, ${ }^{168}$ C. Mills,${ }^{57}$ A. Milov, ${ }^{171}$ D. A. Milstead, ${ }^{146 a, 146 b}$ D. Milstein, ${ }^{171}$ A. A. Minaenko, ${ }^{128}$ M. Miñano, ${ }^{167}$ I. A. Minashvili, ${ }^{65}$ A. I. Mincer, ${ }^{108}$

B. Mindur,${ }^{37}$ M. Mineev, ${ }^{65}$ Y. Ming, ${ }^{130}$ L. M. Mir, ${ }^{11}$ G. Mirabelli, ${ }^{132 a}$ L. Miralles Verge, ${ }^{11}$ A. Misiejuk, ${ }^{76}$ J. Mitrevski, ${ }^{137}$ G. Y. Mitrofanov, ${ }^{128}$ V. A. Mitsou, ${ }^{167}$ S. Mitsui, ${ }^{66}$ P. S. Miyagawa, ${ }^{82}$ K. Miyazaki, ${ }^{67}$ J. U. Mjörnmark, ${ }^{79}$ T. Moa, ${ }^{146 a, 146 b}$ P. Mockett, ${ }^{138}$ S. Moed, ${ }^{57}$ V. Moeller, ${ }^{27}$ K. Mönig, ${ }^{41}$ N. Möser,${ }^{20}$ S. Mohapatra, ${ }^{148}$ B. Mohn, ${ }^{13}$ W. Mohr ${ }^{48}$ S. Mohrdieck-Möck, ${ }^{99}$ A. M. Moisseev, ${ }^{128, a}$ R. Moles-Valls, ${ }^{167}$ J. Molina-Perez, ${ }^{29}$ L. Moneta, ${ }^{49}$ J. Monk,${ }^{77}$ E. Monnier, ${ }^{83}$ S. Montesano, ${ }^{89 a, 89 b}$ F. Monticelli, ${ }^{70}$ S. Monzani, ${ }^{19 a, 19 b}$ R. W. Moore, ${ }^{2}$ G. F. Moorhead, ${ }^{86}$ C. Mora Herrera ${ }^{49}$ A. Moraes, ${ }^{53}$ A. Morais, ${ }^{124 a, c}$ N. Morange, ${ }^{136}$ G. Morello, ${ }^{36 a, 36 b}$ D. Moreno, ${ }^{81}$ M. Moreno Llácer, ${ }^{167}$ P. Morettini, ${ }^{50 a}$ M. Morii,${ }^{57}$ J. Morin, ${ }^{75}$ Y. Morita, ${ }^{66}$ A. K. Morley, ${ }^{29}$ G. Mornacchi, ${ }^{29}$ M-C. Morone, ${ }^{49}$ S. V. Morozov, ${ }^{96}$ J. D. Morris, ${ }^{75}$ H. G. Moser,${ }^{99}$ M. Mosidze,${ }^{51}$ J. Moss, ${ }^{109}$ R. Mount,${ }^{143}$ E. Mountricha, ${ }^{9}$ S. V. Mouraviev, ${ }^{94}$ E. J. W. Moyse,${ }^{84}$ M. Mudrinic, ${ }^{12 b}$ F. Mueller, ${ }^{58 \mathrm{a}}$ J. Mueller, ${ }^{123}$ K. Mueller, ${ }^{20}$ T. A. Müller, ${ }^{98}$ D. Muenstermann, ${ }^{29}$ A. Muijs, ${ }^{105}$ A. Muir, ${ }^{168}$ Y. Munwes, ${ }^{153}$ K. Murakami, ${ }^{66}$ W. J. Murray, ${ }^{129}$ I. Mussche, ${ }^{105}$ E. Musto, ${ }^{102 a, 102 b}$ A. G. Myagkov, ${ }^{128}$ M. Myska, ${ }^{125}$ J. Nadal,${ }^{11}$ K. Nagai, ${ }^{160}$ 
K. Nagano, ${ }^{66}$ Y. Nagasaka, ${ }^{60}$ A. M. Nairz, ${ }^{29}$ Y. Nakahama, ${ }^{115}$ K. Nakamura, ${ }^{155}$ I. Nakano, ${ }^{110}$ G. Nanava, ${ }^{20}$ A. Napier, ${ }^{161}$ M. Nash, ${ }^{77, t}$ N. R. Nation, ${ }^{21}$ T. Nattermann, ${ }^{20}$ T. Naumann, ${ }^{41}$ G. Navarro, ${ }^{162}$ H. A. Neal,${ }^{87}$ E. Nebot ${ }^{80}$ P. Yu. Nechaeva, ${ }^{94}$ A. Negri, ${ }^{19 a, 119 b}$ G. Negri, ${ }^{29}$ S. Nektarijevic, ${ }^{49}$ A. Nelson, ${ }^{64}$ S. Nelson, ${ }^{143}$ T. K. Nelson, ${ }^{143}$ S. Nemecek, ${ }^{125}$ P. Nemethy, ${ }^{108}$ A. A. Nepomuceno, ${ }^{23 a}$ M. Nessi, ${ }^{29, u}$ S. Y. Nesterov, ${ }^{121}$ M. S. Neubauer, ${ }^{165}$ A. Neusiedl, ${ }^{81}$ R. M. Neves, ${ }^{108}$ P. Nevski, ${ }^{24}$ P. R. Newman, ${ }^{17}$ R. B. Nickerson, ${ }^{18}$ R. Nicolaidou, ${ }^{136}$ L. Nicolas, ${ }^{139}$ B. Nicquevert, ${ }^{29}$ F. Niedercorn, ${ }^{115}$ J. Nielsen, ${ }^{137}$ T. Niinikoski, ${ }^{29}$ A. Nikiforov, ${ }^{15}$ V. Nikolaenko, ${ }^{128}$ K. Nikolaev, ${ }^{65}$ I. Nikolic-Audit, ${ }^{78}$ K. Nikolopoulos, ${ }^{24}$ H. Nilsen, ${ }^{48}$ P. Nilsson, ${ }^{7}$ Y. Ninomiya, ${ }^{155}$ A. Nisati, ${ }^{132 a}$ T. Nishiyama, ${ }^{67}$ R. Nisius, ${ }^{99}$ L. Nodulman, ${ }^{5}$ M. Nomachi, ${ }^{116}$ I. Nomidis, ${ }^{154}$ H. Nomoto, ${ }^{155}$ M. Nordberg, ${ }^{29}$ B. Nordkvist, ${ }^{146 a, 146 b}$ P. R. Norton, ${ }^{129}$ J. Novakova, ${ }^{126}$ M. Nozaki, ${ }^{66}$ M. Nožička, ${ }^{41}$ L. Nozka, ${ }^{113}$ I. M. Nugent, ${ }^{159 a}$ A.-E. Nuncio-Quiroz, ${ }^{20}$ G. Nunes Hanninger, ${ }^{20}$ T. Nunnemann, ${ }^{98}$ E. Nurse, ${ }^{77}$ T. Nyman, ${ }^{29}$ B. J. O’Brien, ${ }^{45}$ S. W. O'Neale, ${ }^{17, a}$ D. C. O'Neil, ${ }^{142}$ V. O'Shea, ${ }^{53}$ F. G. Oakham,${ }^{28, e}$ H. Oberlack, ${ }^{99}$ J. Ocariz, ${ }^{78}$ A. Ochi,${ }^{67}$ S. Oda, ${ }^{155}$ S. Odaka, ${ }^{66}$ J. Odier, ${ }^{83}$ H. Ogren, ${ }^{61}$ A. Oh, ${ }^{82}$ S. H. Oh ${ }^{44}$ C. C. Ohm, ${ }^{146 a, 146 b}$ T. Ohshima, ${ }^{101}$ H. Ohshita, ${ }^{140}$ T. K. Ohska ${ }^{66}$ T. Ohsugi, ${ }^{59}$ S. Okada,${ }^{67}$ H. Okawa, ${ }^{163}$ Y. Okumura, ${ }^{101}$ T. Okuyama, ${ }^{155}$ M. Olcese ${ }^{50 a}$ A. G. Olchevski, ${ }^{65}$ M. Oliveira, ${ }^{124 a, h}$ D. Oliveira Damazio, ${ }^{24}$ E. Oliver Garcia, ${ }^{167}$ D. Olivito, ${ }^{120}$ A. Olszewski, ${ }^{38}$ J. Olszowska, ${ }^{38}$ C. Omachi, ${ }^{67}$ A. Onofre, ${ }^{124 a, v}$ P. U.E. Onyisi, ${ }^{30}$ C. J. Oram, ${ }^{159 a}$ M. J. Oreglia, ${ }^{30}$ F. Orellana, ${ }^{49}$ Y. Oren, ${ }^{153}$ D. Orestano, ${ }^{134 a, 134 b}$ I. Orlov, ${ }^{107}$ C. Oropeza Barrera, ${ }^{53}$ R. S. Orr, ${ }^{158}$ E. O. Ortega, ${ }^{130}$ B. Osculati, ${ }^{50 a, 50 b}$ R. Ospanov, ${ }^{120}$ C. Osuna, ${ }^{11}$ G. Otero y Garzon, ${ }^{26}$ J. P Ottersbach, ${ }^{105}$ M. Ouchrif, ${ }^{135 d}$ F. Ould-Saada, ${ }^{117}$ A. Ouraou, ${ }^{136}$ Q. Ouyang, ${ }^{32 \mathrm{a}}$ M. Owen, ${ }^{82}$ S. Owen, ${ }^{139}$ O. K. Øye, ${ }^{13}$ V. E. Ozcan, ${ }^{18 \mathrm{a}}$ N. Ozturk, ${ }^{7}$ A. Pacheco Pages,,${ }^{11}$ C. Padilla Aranda, ${ }^{11}$ E. Paganis, ${ }^{139}$ F. Paige,${ }^{24}$ K. Pajchel, ${ }^{117}$ S. Palestini, ${ }^{29}$ D. Pallin, ${ }^{33}$ A. Palma, ${ }^{124 a, c}$ J. D. Palmer,${ }^{17}$ Y. B. Pan, ${ }^{172}$ E. Panagiotopoulou, ${ }^{9}$ B. Panes,${ }^{31 \mathrm{a}}$ N. Panikashvili, ${ }^{87}$ S. Panitkin,${ }^{24}$ D. Pantea, ${ }^{25 a}$ M. Panuskova, ${ }^{125}$

V. Paolone, ${ }^{123}$ A. Paoloni, ${ }^{133 a, 133 b}$ A. Papadelis, ${ }^{146 a}$ Th. D. Papadopoulou, ${ }^{9}$ A. Paramonov, ${ }^{5}$ W. Park,,${ }^{24, w}$ M. A. Parker, ${ }^{27}$ F. Parodi, ${ }^{50 a, 50 b}$ J. A. Parsons, ${ }^{34}$ U. Parzefall, ${ }^{48}$ E. Pasqualucci, ${ }^{132 a}$ A. Passeri, ${ }^{134 a}$ F. Pastore, ${ }^{134 a, 134 b}$ Fr. Pastore, ${ }^{29}$ G. Pásztor, ${ }^{49, x}$ S. Pataraia, ${ }^{172}$ N. Patel, ${ }^{150}$ J. R. Pater,${ }^{82}$ S. Patricelli, ${ }^{102 a, 102 b}$ T. Pauly,${ }^{29}$ M. Pecsy, ${ }^{144 a}$ M. I. Pedraza Morales, ${ }^{172}$ S. V. Peleganchuk,${ }^{107}$ H. Peng, ${ }^{172}$ R. Pengo, ${ }^{29}$ A. Penson, ${ }^{34}$ J. Penwell, ${ }^{61}$ M. Perantoni, ${ }^{23 a}$ K. Perez,${ }^{34, \mathrm{~s}}$ T. Perez Cavalcanti, ${ }^{41}$ E. Perez Codina, ${ }^{11}$ M. T. Pérez García-Estañ, ${ }^{167}$ V. Perez Reale, ${ }^{34}$ I. Peric,${ }^{20}$ L. Perini, ${ }^{89 a, 89 b}$ H. Pernegger, ${ }^{29}$ R. Perrino, ${ }^{72 a}$ P. Perrodo, ${ }^{4}$ S. Persembe, ${ }^{3 a}$ V. D. Peshekhonov, ${ }^{65}$ O. Peters,${ }^{105}$ B. A. Petersen, ${ }^{29}$ J. Petersen, ${ }^{29}$ T. C. Petersen,${ }^{35}$ E. Petit, ${ }^{83}$ A. Petridis, ${ }^{154}$ C. Petridou, ${ }^{154}$ E. Petrolo, ${ }^{132 a}$ F. Petrucci, ${ }^{134 a, 134 b}$ D. Petschull, ${ }^{41}$ M. Petteni, ${ }^{142}$ R. Pezoa, ${ }^{31 b}$ A. Phan,${ }^{86}$ A. W. Phillips, ${ }^{27}$ P. W. Phillips, ${ }^{129}$ G. Piacquadio, ${ }^{29}$ E. Piccaro, ${ }^{75}$ M. Piccinini, ${ }^{19 a, 19 b}$ A. Pickford, ${ }^{53}$ S. M. Piec,${ }^{41}$ R. Piegaia, ${ }^{26}$ J. E. Pilcher, ${ }^{30}$ A. D. Pilkington, ${ }^{82}$ J. Pina, ${ }^{124 a, c}$ M. Pinamonti, ${ }^{164 a, 164 c}$ A. Pinder, ${ }^{118}$ J. L. Pinfold, ${ }^{2}$ J. Ping, ${ }^{32 c}$ B. Pinto, ${ }^{124 a, c}$ O. Pirotte ${ }^{29}$ C. Pizio, ${ }^{89 a, 89 b}$ R. Placakyte, ${ }^{41}$ M. Plamondon, ${ }^{169}$ W. G. Plano,${ }^{82}$ M.-A. Pleier, ${ }^{24}$ A. V. Pleskach, ${ }^{128}$ A. Poblaguev,${ }^{24}$ S. Poddar, ${ }^{58 a}$ F. Podlyski,${ }^{33}$ L. Poggioli, ${ }^{115}$ T. Poghosyan,${ }^{20}$ M. Pohl,${ }^{49}$ F. Polci, ${ }^{55}$ G. Polesello, ${ }^{119 a}$ A. Policicchio, ${ }^{138}$ A. Polini, ${ }^{19 a}$ J. Poll, ${ }^{75}$ V. Polychronakos, ${ }^{24}$ D. M. Pomarede, ${ }^{136}$ D. Pomeroy, ${ }^{22}$ K. Pommès ${ }^{29}$

L. Pontecorvo, ${ }^{132 a}$ B. G. Pope,${ }^{88}$ G. A. Popeneciu, ${ }^{25 a}$ D. S. Popovic, ${ }^{12 a}$ A. Poppleton, ${ }^{29}$ X. Portell Bueso, ${ }^{48}$ R. Porter, ${ }^{163}$ C. Posch, ${ }^{21}$ G. E. Pospelov, ${ }^{99}$ S. Pospisil, ${ }^{127}$ I. N. Potrap, ${ }^{99}$ C. J. Potter, ${ }^{149}$ C. T. Potter, ${ }^{114}$ G. Poulard, ${ }^{29}$ J. Poveda, ${ }^{172}$ R. Prabhu, ${ }^{77}$ P. Pralavorio, ${ }^{83}$ S. Prasad,${ }^{57}$ R. Pravahan, ${ }^{7}$ S. Prell,${ }^{64}$ K. Pretzl,${ }^{16}$ L. Pribyl,${ }^{29}$ D. Price, ${ }^{61}$ L.E. Price, ${ }^{5}$ M. J. Price, ${ }^{29}$ P. M. Prichard,${ }^{73}$ D. Prieur, ${ }^{123}$ M. Primavera, ${ }^{72 a}$ K. Prokofiev, ${ }^{108}$ F. Prokoshin,,${ }^{31 b}$

S. Protopopescu, ${ }^{24}$ J. Proudfoot,${ }^{5}$ X. Prudent, ${ }^{43}$ H. Przysiezniak, ${ }^{4}$ S. Psoroulas, ${ }^{20}$ E. Ptacek, ${ }^{114}$ J. Purdham, ${ }^{87}$

M. Purohit,${ }^{24, w}$ P. Puzo, ${ }^{115}$ Y. Pylypchenko, ${ }^{117}$ J. Qian, ${ }^{87}$ Z. Qian, ${ }^{83}$ Z. Qin,${ }^{41}$ A. Quadt, ${ }^{54}$ D. R. Quarrie, ${ }^{14}$ W. B. Quayle, ${ }^{172}$ F. Quinonez, ${ }^{31 \mathrm{a}}$ M. Raas, ${ }^{104}$ V. Radescu, ${ }^{58 \mathrm{~b}}$ B. Radics,${ }^{20}$ T. Rador, ${ }^{18 \mathrm{a}}$ F. Ragusa, ${ }^{89 \mathrm{a}, 89 \mathrm{~b}}$ G. Rahal, ${ }^{177}$

A. M. Rahimi, ${ }^{109}$ D. Rahm, ${ }^{24}$ S. Rajagopalan, ${ }^{24}$ M. Rammensee, ${ }^{48}$ M. Rammes, ${ }^{141}$ M. Ramstedt, ${ }^{146 a, 146 b}$ K. Randrianarivony, ${ }^{28}$ P. N. Ratoff, ${ }^{71}$ F. Rauscher, ${ }^{98}$ E. Rauter, ${ }^{99}$ M. Raymond,${ }^{29}$ A. L. Read,,${ }^{117}$ D. M. Rebuzzi, ${ }^{119 a, 119 b}$ A. Redelbach, ${ }^{173}$ G. Redlinger, ${ }^{24}$ R. Reece, ${ }^{120}$ K. Reeves, ${ }^{40}$ A. Reichold, ${ }^{105}$ E. Reinherz-Aronis, ${ }^{153}$ A. Reinsch, ${ }^{114}$ I. Reisinger, ${ }^{42}$ D. Reljic, ${ }^{12 a}$ C. Rembser, ${ }^{29}$ Z. L. Ren, ${ }^{151}$ A. Renaud,${ }^{115}$ P. Renkel, ${ }^{39}$ B. Rensch, ${ }^{35}$ M. Rescigno, ${ }^{132 a}$ S. Resconi, ${ }^{89 a}$ B. Resende, ${ }^{136}$ P. Reznicek, ${ }^{98}$ R. Rezvani, ${ }^{158}$

A. Richards, ${ }^{77}$ R. Richter, ${ }^{99}$ E. Richter-Was, ${ }^{38, y}$ M. Ridel,,${ }^{78}$ S. Rieke ${ }^{81}$ M. Rijpstra, ${ }^{105}$ M. Rijssenbeek,,${ }^{148}$ A. Rimoldi, ${ }^{119 a, 119 b}$ L. Rinaldi, ${ }^{19 a}$ R. R. Rios, ${ }^{39}$ I. Riu, ${ }^{11}$ G. Rivoltella, ${ }^{89 a, 89 b}$ F. Rizatdinova, ${ }^{112}$ E. Rizvi, ${ }^{75}$ S. H. Robertson, ${ }^{85, j}$ A. Robichaud-Veronneau, ${ }^{49}$ D. Robinson, ${ }^{27}$ J.E. M. Robinson, ${ }^{77}$ M. Robinson, ${ }^{114}$ A. Robson, ${ }^{53}$ J. G. Rocha de Lima, ${ }^{106}$ C. Roda, ${ }^{122 a, 122 b}$ D. Roda Dos Santos, ${ }^{29}$ S. Rodier, ${ }^{80}$ D. Rodriguez, ${ }^{162}$

Y. Rodriguez Garcia, ${ }^{15}$ A. Roe,${ }^{54}$ S. Roe,${ }^{29}$ O. Røhne, ${ }^{117}$ V. Rojo, ${ }^{1}$ S. Rolli, ${ }^{161}$ A. Romaniouk, ${ }^{96}$ V. M. Romanov ${ }^{65}$ G. Romeo, ${ }^{26}$ D. Romero Maltrana, ${ }^{31 \mathrm{a}}$ L. Roos, ${ }^{78}$ E. Ros, ${ }^{167}$ S. Rosati, ${ }^{132 \mathrm{a}, 132 \mathrm{~b}}$ M. Rose, ${ }^{76}$ G. A. Rosenbaum, ${ }^{158}$ 
E. I. Rosenberg, ${ }^{64}$ P. L. Rosendahl, ${ }^{13}$ L. Rosselet, ${ }^{49}$ V. Rossetti, ${ }^{11}$ E. Rossi, ${ }^{102 a, 102 b}$ L. P. Rossi, ${ }^{50 a}$ L. Rossi, ${ }^{89 a, 89 b}$ M. Rotaru, ${ }^{25 a}$ I. Roth, ${ }^{171}$ J. Rothberg, ${ }^{138}$ D. Rousseau, ${ }^{115}$ C. R. Royon, ${ }^{136}$ A. Rozanov ${ }^{83}$ Y. Rozen, ${ }^{152}$ X. Ruan, ${ }^{115}$ I. Rubinskiy, ${ }^{41}$ B. Ruckert ${ }^{98}$ N. Ruckstuhl, ${ }^{105}$ V. I. Rud,${ }^{97}$ G. Rudolph, ${ }^{62}$ F. Rühr, ${ }^{6}$ F. Ruggieri,,${ }^{134 a, 134 b}$ A. Ruiz-Martinez, ${ }^{64}$ E. Rulikowska-Zarebska, ${ }^{37}$ V. Rumiantsev, ${ }^{91, a}$ L. Rumyantsev, ${ }^{65}$ K. Runge, ${ }^{48}$ O. Runolfsson, ${ }^{20}$ Z. Rurikova, ${ }^{48}$ N. A. Rusakovich, ${ }^{65}$ D. R. Rust, ${ }^{61}$ J. P. Rutherfoord, ${ }^{6}$ C. Ruwiedel, ${ }^{14}$ P. Ruzicka, ${ }^{125}$ Y. F. Ryabov, ${ }^{121}$ V. Ryadovikov, ${ }^{128}$ P. Ryan, ${ }^{88}$ M. Rybar, ${ }^{126}$ G. Rybkin, ${ }^{15}$ N. C. Ryder, ${ }^{118}$ S. Rzaeva, ${ }^{10}$ A. F. Saavedra, ${ }^{150}$ I. Sadeh, ${ }^{153}$ H. F-W. Sadrozinski, ${ }^{137}$ R. Sadykov, ${ }^{65}$ F. Safai Tehrani, ${ }^{132 a, 132 b}$ H. Sakamoto, ${ }^{155}$ G. Salamanna, ${ }^{105}$ A. Salamon, ${ }^{133 a}$ M. Saleem, ${ }^{111}$ D. Salihagic, ${ }^{99}$ A. Salnikov, ${ }^{143}$ J. Salt, ${ }^{167}$ B. M. Salvachua Ferrando, ${ }^{5}$ D. Salvatore, ${ }^{36 a, 36 b}$ F. Salvatore, ${ }^{149}$ A. Salzburger ${ }^{29}$ D. Sampsonidis, ${ }^{154}$ B. H. Samset,${ }^{117}$ H. Sandaker, ${ }^{13}$ H. G. Sander,${ }^{81}$ M. P. Sanders,${ }^{98}$ M. Sandhoff, ${ }^{174}$ P. Sandhu, ${ }^{158}$ T. Sandoval,,${ }^{27}$ R. Sandstroem, ${ }^{105}$ S. Sandvoss,${ }^{174}$ D. P. C. Sankey, ${ }^{129}$ A. Sansoni, ${ }^{47}$ C. Santamarina Rios, ${ }^{85}$ C. Santoni,${ }^{33}$ R. Santonico, ${ }^{133 a, 133 b}$ H. Santos, ${ }^{124 a}$ J. G. Saraiva, ${ }^{124 a, c}$ T. Sarangi, ${ }^{172}$ E. Sarkisyan-Grinbaum, ${ }^{7}$ F. Sarri, ${ }^{122 a, 122 b}$ G. Sartisohn, ${ }^{174}$ O. Sasaki, ${ }^{66}$ T. Sasaki, ${ }^{66}$ N. Sasao, ${ }^{68}$ I. Satsounkevitch, ${ }^{90}$ G. Sauvage, ${ }^{4}$ J. B. Sauvan, ${ }^{115}$ P. Savard,${ }^{158, e}$ V. Savinov, ${ }^{123}$ D. O. Savu, ${ }^{29}$ P. Savva, ${ }^{9}$ L. Sawyer,${ }^{24, k}$ D. H. Saxon, ${ }^{53}$ L. P. Says, ${ }^{33}$ C. Sbarra, ${ }^{19 a, 19 b}$ A. Sbrizzi, ${ }^{19 a, 19 b}$ O. Scallon, ${ }^{93}$ D. A. Scannicchio, ${ }^{163}$ J. Schaarschmidt, ${ }^{115}$ P. Schacht, ${ }^{99}$ U. Schäfer, ${ }^{81}$ S. Schaepe, ${ }^{20}$ S. Schaetzel, ${ }^{58 b}$ A. C. Schaffer, ${ }^{115}$ D. Schaile, ${ }^{98}$ R. D. Schamberger, ${ }^{148}$ A. G. Schamov, ${ }^{107}$ V. Scharf, ${ }^{58 \mathrm{a}}$ V. A. Schegelsky, ${ }^{121}$ D. Scheirich,${ }^{87}$ M. I. Scherzer, ${ }^{14}$ C. Schiavi, ${ }^{50 a, 50 b}$ J. Schieck,${ }^{98}$ M. Schioppa,${ }^{36 a, 36 b}$ S. Schlenker, ${ }^{29}$ J. L. Schlereth, ${ }^{5}$ E. Schmidt, ${ }^{48}$ M. P. Schmidt, ${ }^{175, a}$ K. Schmieden, ${ }^{20}$ C. Schmitt, ${ }^{81}$ M. Schmitz, ${ }^{20}$ A. Schöning, ${ }^{58 b}$ M. Schott, ${ }^{29}$ D. Schouten, ${ }^{142}$ J. Schovancova, ${ }^{125}$ M. Schram, ${ }^{85}$ C. Schroeder, ${ }^{81}$ N. Schroer, ${ }^{58 c}$ S. Schuh, ${ }^{29}$ G. Schuler, ${ }^{29}$ J. Schultes, ${ }^{174}$ H.-C. Schultz-Coulon, ${ }^{58 a}$ H. Schulz, ${ }^{15}$ J. W. Schumacher, ${ }^{20}$

M. Schumacher, ${ }^{48}$ B. A. Schumm, ${ }^{137} \mathrm{Ph}$. Schune, ${ }^{136}$ C. Schwanenberger, ${ }^{82}$ A. Schwartzman, ${ }^{143} \mathrm{Ph}$. Schwemling ${ }^{78}$

R. Schwienhorst ${ }^{88}$ R. Schwierz, ${ }^{43}$ J. Schwindling, ${ }^{136}$ W. G. Scott, ${ }^{129}$ J. Searcy, ${ }^{114}$ E. Sedykh, ${ }^{121}$ E. Segura, ${ }^{11}$ S. C. Seidel,${ }^{103}$ A. Seiden, ${ }^{137}$ F. Seifert, ${ }^{43}$ J. M. Seixas, ${ }^{23 a}$ G. Sekhniaidze, ${ }^{102 a}$ D. M. Seliverstov, ${ }^{121}$ B. Sellden, ${ }^{146 a}$ G. Sellers, ${ }^{73}$ M. Seman, ${ }^{144 b}$ N. Semprini-Cesari, ${ }^{19 a, 19 b}$ C. Serfon, ${ }^{98}$ L. Serin, ${ }^{115}$ R. Seuster, ${ }^{99}$ H. Severini, ${ }^{111}$ M. E. Sevior, ${ }^{86}$ A. Sfyrla, ${ }^{29}$ E. Shabalina, ${ }^{54}$ M. Shamim,,${ }^{114}$ L. Y. Shan, ${ }^{32 a}$ J. T. Shank,${ }^{21}$ Q. T. Shao, ${ }^{86}$ M. Shapiro, ${ }^{14}$ P. B. Shatalov, ${ }^{95}$ L. Shaver, ${ }^{6}$ C. Shaw,${ }^{53}$ K. Shaw, ${ }^{164 a, 164 c}$ D. Sherman, ${ }^{175}$ P. Sherwood, ${ }^{77}$ A. Shibata, ${ }^{108}$ S. Shimizu, ${ }^{29}$ M. Shimojima, ${ }^{100}$ T. Shin, ${ }^{56}$ A. Shmeleva, ${ }^{94}$ M. J. Shochet,${ }^{30}$ D. Short, ${ }^{118}$ M. A. Shupe,${ }^{6}$ P. Sicho, ${ }^{125}$ A. Sidoti, ${ }^{132 a, 132 b}$ A. Siebel, ${ }^{174}$ F. Siegert, ${ }^{48}$ J. Siegrist, ${ }^{14}$ Dj. Sijacki, ${ }^{12 a}$ O. Silbert, ${ }^{171}$ J. Silva, ${ }^{124 a, c}$ Y. Silver, ${ }^{153}$ D. Silverstein, ${ }^{143}$ S. B. Silverstein, ${ }^{146 a}$ V. Simak, ${ }^{127}$ O. Simard, ${ }^{136}$ Lj. Simic, ${ }^{12 a}$ S. Simion, ${ }^{115}$ B. Simmons, ${ }^{77}$ M. Simonyan, ${ }^{35}$ P. Sinervo, ${ }^{158}$ N. B. Sinev, ${ }^{114}$ V. Sipica, ${ }^{141}$ G. Siragusa, ${ }^{81}$ A. N. Sisakyan, ${ }^{65}$ S. Yu. Sivoklokov, ${ }^{97}$ J. Sjölin, ${ }^{146 a, 146 b}$ T. B. Sjursen, ${ }^{13}$ L. A. Skinnari, ${ }^{14}$ K. Skovpen, ${ }^{107}$ P. Skubic,,${ }^{11}$ N. Skvorodnev, ${ }^{22}$ M. Slater,${ }^{17}$

T. Slavicek, ${ }^{127}$ K. Sliwa, ${ }^{161}$ T. J. Sloan, ${ }^{71}$ J. Sloper ${ }^{29}$ V. Smakhtin, ${ }^{171}$ S. Yu. Smirnov,${ }^{96}$ L. N. Smirnova, ${ }^{97}$ O. Smirnova, ${ }^{79}$ B. C. Smith, ${ }^{57}$ D. Smith, ${ }^{143}$ K. M. Smith, ${ }^{53}$ M. Smizanska, ${ }^{71}$ K. Smolek, ${ }^{127}$ A. A. Snesarev, ${ }^{94}$ S. W. Snow, ${ }^{82}$ J. Snow, ${ }^{111}$ J. Snuverink, ${ }^{105}$ S. Snyder, ${ }^{24}$ M. Soares, ${ }^{124 a}$ R. Sobie, ${ }^{169, j}$ J. Sodomka, ${ }^{127}$ A. Soffer, ${ }^{153}$ C. A. Solans, ${ }^{167}$ M. Solar, ${ }^{127}$ J. Solc, ${ }^{127}$ E. Soldatov, ${ }^{96}$ U. Soldevila, ${ }^{167}$ E. Solfaroli Camillocci, ${ }^{132 a, 132 b}$ A. A. Solodkov, ${ }^{128}$ O. V. Solovyanov, ${ }^{128}$ J. Sondericker, ${ }^{24}$ N. Soni, ${ }^{2}$ V. Sopko, ${ }^{127}$ B. Sopko, ${ }^{127}$ M. Sorbi, ${ }^{89 a, 89 b}$ M. Sosebee, ${ }^{7}$ A. Soukharev, ${ }^{107}$ S. Spagnolo, ${ }^{72 a, 72 b}$ F. Spanò,${ }^{34}$ R. Spighi, ${ }^{19 a}$ G. Spigo, ${ }^{29}$ F. Spila,,${ }^{132 a, 132 b}$ E. Spiriti, ${ }^{134 a}$ R. Spiwoks, ${ }^{29}$ M. Spousta, ${ }^{126}$ T. Spreitzer ${ }^{158}$ B. Spurlock, ${ }^{7}$ R. D. St. Denis, ${ }^{53}$ T. Stahl,${ }^{141}$ J. Stahlman, ${ }^{120}$ R. Stamen, ${ }^{58 a}$ E. Stanecka ${ }^{29}$ R. W. Stanek, ${ }^{5}$ C. Stanescu, ${ }^{134 a}$ S. Stapnes, ${ }^{117}$ E. A. Starchenko, ${ }^{128}$ J. Stark,${ }^{55}$ P. Staroba,${ }^{125}$ P. Starovoitov,${ }^{91}$ A. Staude, ${ }^{98}$ P. Stavina,${ }^{144 a}$ G. Stavropoulos, ${ }^{14}$ G. Steele,${ }^{53}$ P. Steinbach,${ }^{43}$ P. Steinberg, ${ }^{24}$ I. Stekl, ${ }^{127}$ B. Stelzer, ${ }^{142}$ H. J. Stelzer, ${ }^{41}$ O. Stelzer-Chilton, ${ }^{159 a}$ H. Stenzel,${ }^{52}$ K. Stevenson, ${ }^{75}$ G. A. Stewart, ${ }^{53}$ J. A. Stillings, ${ }^{20}$ T. Stockmanns, ${ }^{20}$ M. C. Stockton, ${ }^{29}$ K. Stoerig, ${ }^{48}$ G. Stoicea, ${ }^{25 a}$ S. Stonjek, ${ }^{99}$ P. Strachota, ${ }^{126}$ A. R. Stradling, ${ }^{7}$ A. Straessner, ${ }^{43}$ J. Strandberg, ${ }^{87}$ S. Strandberg, ${ }^{146 a, 146 b}$ A. Strandlie, ${ }^{117}$ M. Strang, ${ }^{109}$ E. Strauss, ${ }^{143}$ M. Strauss, ${ }^{111}$ P. Strizenec, ${ }^{144 b}$ R. Ströhmer, ${ }^{173}$ D. M. Strom, ${ }^{114}$ J. A. Strong, ${ }^{76, a}$ R. Stroynowski, ${ }^{39}$ J. Strube, ${ }^{129}$ B. Stugu, ${ }^{13}$ I. Stumer, ${ }^{24, a}$ J. Stupak, ${ }^{148}$ P. Sturm, ${ }^{174}$ D. A. Soh, ${ }^{151, p}$ D. Su, ${ }^{143}$ HS. Subramania, ${ }^{2}$ A. Succurro, ${ }^{11}$ Y. Sugaya, ${ }^{116}$ T. Sugimoto, ${ }^{101}$ C. Suhr, ${ }^{106}$ K. Suita, ${ }^{67}$ M. Suk, ${ }^{126}$ V. V. Sulin, ${ }^{94}$ S. Sultansoy, ${ }^{3 \mathrm{~d}}$ T. Sumida, ${ }^{29}$ X. Sun, ${ }^{55}$ J. E. Sundermann, ${ }^{48}$ K. Suruliz, ${ }^{164 a, 164 b}$ S. Sushkov, ${ }^{11}$ G. Susinno, ${ }^{36 a, 36 b}$

M. R. Sutton, ${ }^{139}$ Y. Suzuki, ${ }^{66}$ Yu. M. Sviridov, ${ }^{128}$ S. Swedish, ${ }^{168}$ I. Sykora, ${ }^{144 a}$ T. Sykora, ${ }^{126}$ B. Szeless, ${ }^{29}$ J. Sánchez, ${ }^{167}$ D. Ta, ${ }^{105}$ K. Tackmann, ${ }^{29}$ A. Taffard, ${ }^{163}$ R. Tafirout, ${ }^{159 a}$ A. Taga, ${ }^{117}$ N. Taiblum, ${ }^{153}$ Y. Takahashi, ${ }^{101}$ H. Takai,${ }^{24}$ R. Takashima, ${ }^{69}$ H. Takeda,${ }^{67}$ T. Takeshita, ${ }^{140}$ M. Talby, ${ }^{83}$ A. Talyshev, ${ }^{107}$ M. C. Tamsett, ${ }^{24}$ J. Tanaka,${ }^{155}$ R. Tanaka, ${ }^{115}$ S. Tanaka, ${ }^{131}$ S. Tanaka, ${ }^{66}$ Y. Tanaka, ${ }^{100}$ K. Tani,${ }^{67}$ N. Tannoury,${ }^{83}$ G. P. Tappern,${ }^{29}$ S. Tapprogge, ${ }^{81}$ D. Tardif, ${ }^{158}$ S. Tarem, ${ }^{152}$ F. Tarrade,${ }^{24}$ G. F. Tartarelli, ${ }^{89 a}$ P. Tas, ${ }^{126}$ M. Tasevsky, ${ }^{125}$ E. Tassi, ${ }^{36 a, 36 b}$ M. Tatarkhanov, ${ }^{14}$ 
C. Taylor, ${ }^{77}$ F. E. Taylor, ${ }^{92}$ G. N. Taylor, ${ }^{86}$ W. Taylor, ${ }^{159 b}$ M. Teixeira Dias Castanheira, ${ }^{75}$ P. Teixeira-Dias, ${ }^{76}$ K. K. Temming ${ }^{48}$ H. Ten Kate, ${ }^{29}$ P. K. Teng, ${ }^{151}$ S. Terada,${ }^{66}$ K. Terashi, ${ }^{155}$ J. Terron ${ }^{80}$ M. Terwort, ${ }^{41, n}$ M. Testa, ${ }^{47}$ R. J. Teuscher, ${ }^{158, j}$ C. M. Tevlin, ${ }^{82}$ J. Thadome, ${ }^{174}$ J. Therhaag, ${ }^{20}$ T. Theveneaux-Pelzer, ${ }^{78}$ M. Thioye,${ }^{175}$ S. Thoma, ${ }^{48}$ J. P. Thomas, ${ }^{17}$ E. N. Thompson, ${ }^{84}$ P. D. Thompson, ${ }^{17}$ P. D. Thompson, ${ }^{158}$ A. S. Thompson, ${ }^{53}$ E. Thomson, ${ }^{120}$

M. Thomson, ${ }^{27}$ R. P. Thun, ${ }^{87}$ T. Tic,,${ }^{125}$ V. O. Tikhomirov, ${ }^{94}$ Y. A. Tikhonov, ${ }^{107}$ C. J. W. P. Timmermans,,${ }^{104}$ P. Tipton, ${ }^{175}$ F. J. Tique Aires Viegas, ${ }^{29} \mathrm{~S}$. Tisserant,${ }^{83} \mathrm{~J}$. Tobias, ${ }^{48} \mathrm{~B}$. Toczek, ${ }^{37}$ T. Todorov, ${ }^{4} \mathrm{~S}$. Todorova-Nova, ${ }^{161}$ B. Toggerson, ${ }^{163}$ J. Tojo, ${ }^{66} \mathrm{~S}$. Tokár, ${ }^{144 a} \mathrm{~K}$. Tokunaga,${ }^{67} \mathrm{~K}$. Tokushuku, ${ }^{66} \mathrm{~K}$. Tollefson, ${ }^{88} \mathrm{M}$. Tomoto, ${ }^{101}$ L. Tompkins, ${ }^{14}$ K. Toms, ${ }^{103}$ G. Tong, ${ }^{32 a}$ A. Tonoyan, ${ }^{13}$ C. Topfel,${ }^{16}$ N. D. Topilin, ${ }^{65}$ I. Torchiani, ${ }^{29}$ E. Torrence,${ }^{114}$ E. Torró Pastor, ${ }^{167}$ J. Toth ${ }^{83, x}$ F. Touchard ${ }^{83}$ D. R. Tovey, ${ }^{139}$ D. Traynor, ${ }^{75}$ T. Trefzger, ${ }^{173}$ J. Treis,${ }^{20}$ L. Tremblet, ${ }^{29}$ A. Tricoli, ${ }^{29}$ I. M. Trigger, ${ }^{159 a}$ S. Trincaz-Duvoid, ${ }^{78}$ T. N. Trinh,${ }^{78}$ M. F. Tripiana,${ }^{70}$ N. Triplett, ${ }^{64}$ W. Trischuk,${ }^{158}$

A. Trivedi,${ }^{24, w}$ B. Trocmé,${ }^{55}$ C. Troncon, ${ }^{89 a}$ M. Trottier-McDonald, ${ }^{142}$ A. Trzupek, ${ }^{38}$ C. Tsarouchas,${ }^{29}$

J. C-L. Tseng, ${ }^{118}$ M. Tsiakiris, ${ }^{105}$ P. V. Tsiareshka, ${ }^{90}$ D. Tsionou, ${ }^{4}$ G. Tsipolitis, ${ }^{9}$ V. Tsiskaridze,${ }^{48}$

E. G. Tskhadadze,${ }^{51}$ I. I. Tsukerman,${ }^{95}$ V. Tsulaia, ${ }^{123}$ J.-W. Tsung, ${ }^{20}$ S. Tsuno, ${ }^{66}$ D. Tsybychev,${ }^{148}$ A. Tua, ${ }^{139}$ J. M. Tuggle,${ }^{30}$ M. Turala, ${ }^{38}$ D. Turecek, ${ }^{127}$ I. Turk Cakir, ${ }^{3 e}$ E. Turlay, ${ }^{105}$ R. Turra,${ }^{89 a, 89 b}$ P. M. Tuts, ${ }^{34}$ A. Tykhonov, ${ }^{74}$ M. Tylmad, ${ }^{146 a, 146 b}$ M. Tyndel, ${ }^{129}$ H. Tyrvainen, ${ }^{29}$ G. Tzanakos,${ }^{8}$ K. Uchida, ${ }^{20}$ I. Ueda, ${ }^{155}$ R. Ueno,${ }^{28}$ M. Ugland, ${ }^{13}$ M. Uhlenbrock ${ }^{20}$ M. Uhrmacher, ${ }^{54}$ F. Ukegawa, ${ }^{160}$ G. Unal, ${ }^{29}$ D. G. Underwood,${ }^{5}$ A. Undrus,${ }^{24}$ G. Unel, ${ }^{163}$ Y. Unno, ${ }^{66}$ D. Urbaniec, ${ }^{34}$ E. Urkovsky, ${ }^{153}$ P. Urrejola, ${ }^{31 a}$ G. Usai, ${ }^{7}$ M. Uslenghi, ${ }^{19 a, 119 b}$ L. Vacavant, ${ }^{83}$ V. Vacek, ${ }^{127}$ B. Vachon, ${ }^{85} \mathrm{~S}$. Vahsen, ${ }^{14} \mathrm{C}$. Valderanis, ${ }^{99} \mathrm{~J}$. Valenta, ${ }^{125} \mathrm{P}$. Valente, ${ }^{132 \mathrm{a}} \mathrm{S}$. Valentinetti, ${ }^{19 \mathrm{a}, 19 \mathrm{~b}} \mathrm{~S}$. Valkar, ${ }^{126}$ E. Valladolid Gallego, ${ }^{167}$ S. Vallecorsa, ${ }^{152}$ J. A. Valls Ferrer, ${ }^{167}$ H. van der Graaf, ${ }^{105}$ E. van der Kraaij, ${ }^{105}$ R. Van Der Leeuw, ${ }^{105}$ E. van der Poel, ${ }^{105}$ D. van der Ster, ${ }^{29}$ B. Van Eijk, ${ }^{105}$ N. van Eldik,${ }^{84}$ P. van Gemmeren, ${ }^{5}$ Z. van Kesteren, ${ }^{105}$ I. van Vulpen, ${ }^{105}$ W. Vandelli, ${ }^{29}$ G. Vandoni, ${ }^{29}$ A. Vaniachine, ${ }^{5}$ P. Vankov,${ }^{41}$ F. Vannucci, ${ }^{78}$

F. Varela Rodriguez, ${ }^{29}$ R. Vari, ${ }^{132 a}$ E. W. Varnes, ${ }^{6}$ D. Varouchas, ${ }^{14}$ A. Vartapetian, ${ }^{7}$ K. E. Varvell, ${ }^{150}$ V. I. Vassilakopoulos ${ }^{56}$ F. Vazeille, ${ }^{33}$ G. Vegni,${ }^{89 a, 89 b}$ J. J. Veillet, ${ }^{115}$ C. Vellidis, ${ }^{8}$ F. Veloso,${ }^{124 a}$ R. Veness,${ }^{29}$ S. Veneziano, ${ }^{132 a}$ A. Ventura, ${ }^{72 a, 72 b}$ D. Ventura, ${ }^{138}$ M. Venturi, ${ }^{48}$ N. Venturi, ${ }^{16}$ V. Vercesi, ${ }^{119 a}$ M. Verducci, ${ }^{138}$ W. Verkerke, ${ }^{105}$ J. C. Vermeulen, ${ }^{105}$ A. Vest,${ }^{43}$ M.C. Vetterli, ${ }^{142, e}$ I. Vichou, ${ }^{165}$ T. Vickey, ${ }^{145 b, z}$ G. H. A. Viehhauser, ${ }^{18}$ S. Viel, ${ }^{168}$ M. Villa, ${ }^{19 a, 19 b}$ M. Villaplana Perez,${ }^{167}$ E. Vilucchi, ${ }^{47}$ M. G. Vincter, ${ }^{28}$ E. Vinek, ${ }^{29}$ V. B. Vinogradov, ${ }^{65}$ M. Virchaux, ${ }^{136, a}$ S. Viret,${ }^{33}$ J. Virzi, ${ }^{14}$ A. Vitale, ${ }^{19 a, 19 b}$ O. Vitells ${ }^{171}$ M. Viti,${ }^{41}$ I. Vivarelli, ${ }^{48}$ F. Vives Vaque, ${ }^{11}$ S. Vlachos, ${ }^{9}$ M. Vlasak,${ }^{127}$ N. Vlasov,${ }^{20}$ A. Vogel,${ }^{20}$ P. Vokac,,${ }^{127}$ G. Volpi,${ }^{47}$ M. Volpi,${ }^{11}$ G. Volpini, ${ }^{89 a}$ H. von der Schmitt,,${ }^{99}$ J. von Loeben, ${ }^{99}$ H. von Radziewski, ${ }^{48}$ E. von Toerne, ${ }^{20}$ V. Vorobel, ${ }^{126}$ A. P. Vorobiev, ${ }^{128}$ V. Vorwerk, ${ }^{11}$ M. Vos, ${ }^{167}$ R. Voss,${ }^{29}$ T. T. Voss,${ }^{174}$ J. H. Vossebeld, ${ }^{73}$ A. S. Vovenko, ${ }^{128}$ N. Vranjes, ${ }^{12 a}$ M. Vranjes Milosavljevic, ${ }^{12 a}$ V. Vrba, ${ }^{125}$ M. Vreeswijk, ${ }^{105} \mathrm{~T}$. Vu Anh, ${ }^{81}$ R. Vuillermet, ${ }^{29}$ I. Vukotic, ${ }^{115}$ W. Wagner, ${ }^{174}$ P. Wagner, ${ }^{120}$ H. Wahlen, ${ }^{174}$ J. Wakabayashi, ${ }^{101}$ J. Walbersloh,${ }^{42}$ S. Walch ${ }^{87}$ J. Walder, ${ }^{71}$ R. Walker,${ }^{98}$

W. Walkowiak, ${ }^{141}$ R. Wall, ${ }^{175}$ P. Waller,${ }^{73}$ C. Wang,,${ }^{44}$ H. Wang, ${ }^{172}$ H. Wang, ${ }^{32 b}$ J. Wang, ${ }^{151}$ J. Wang, ${ }^{32 \mathrm{~d}}$ J. C. Wang, ${ }^{138}$ R. Wang, ${ }^{103}$ S. M. Wang, ${ }^{151}$ A. Warburton, ${ }^{85}$ C. P. Ward, ${ }^{27}$ M. Warsinsky, ${ }^{48}$ P. M. Watkins,${ }^{17}$ A. T. Watson, ${ }^{17}$ M. F. Watson, ${ }^{17}$ G. Watts, ${ }^{138}$ S. Watts, ${ }^{82}$ A. T. Waugh,${ }^{150}$ B. M. Waugh, ${ }^{77}$ J. Weber,${ }^{42}$ M. Weber, ${ }^{129}$ M. S. Weber ${ }^{16}$ P. Weber, ${ }^{54}$ A. R. Weidberg, ${ }^{118}$ P. Weigell, ${ }^{99}$ J. Weingarten, ${ }^{54}$ C. Weiser, ${ }^{48}$ H. Wellenstein, ${ }^{22}$ P. S. Wells,${ }^{29}$ M. Wen, ${ }^{47}$ T. Wenaus, ${ }^{24}$ S. Wendler, ${ }^{123}$ Z. Weng, ${ }^{151, p}$ T. Wengler, ${ }^{29}$ S. Wenig, ${ }^{29}$ N. Wermes, ${ }^{20}$ M. Werner, ${ }^{48}$ P. Werner, ${ }^{29}$ M. Werth, ${ }^{163}$ M. Wessels, ${ }^{58 a} \mathrm{~K}$. Whalen ${ }^{28}$ S. J. Wheeler-Ellis, ${ }^{163}$ S. P. Whitaker, ${ }^{21}$ A. White, ${ }^{7}$ M. J. White, ${ }^{86}$ S. White, ${ }^{24}$ S. R. Whitehead, ${ }^{118}$ D. Whiteson, ${ }^{163}$ D. Whittington, ${ }^{61}$ F. Wicek,,${ }^{15}$

D. Wicke, ${ }^{174}$ F. J. Wickens, ${ }^{129}$ W. Wiedenmann, ${ }^{172}$ M. Wielers, ${ }^{129}$ P. Wienemann, ${ }^{20}$ C. Wiglesworth, ${ }^{73}$

L. A. M. Wiik, ${ }^{48}$ P. A. Wijeratne, ${ }^{77}$ A. Wildauer, ${ }^{167}$ M. A. Wildt, ${ }^{41, n}$ I. Wilhelm, ${ }^{126}$ H. G. Wilkens, ${ }^{29}$ J. Z. Will, ${ }^{98}$ E. Williams, ${ }^{34}$ H. H. Williams, ${ }^{120}$ W. Willis, ${ }^{34}$ S. Willocq, ${ }^{84}$ J. A. Wilson, ${ }^{17}$ M. G. Wilson, ${ }^{143}$ A. Wilson, ${ }^{87}$ I. Wingerter-Seez, ${ }^{4}$ S. Winkelmann, ${ }^{48}$ F. Winklmeier, ${ }^{29}$ M. Wittgen, ${ }^{143}$ M. W. Wolter, ${ }^{38}$ H. Wolters, ${ }^{124 a, h}$ G. Wooden, ${ }^{118}$ B. K. Wosiek, ${ }^{38}$ J. Wotschack, ${ }^{29}$ M. J. Woudstra, ${ }^{84}$ K. Wraight, ${ }^{53}$ C. Wright, ${ }^{53}$ B. Wrona, ${ }^{73}$ S. L. Wu, ${ }^{172}$ X. Wu ${ }^{49}$ Y. Wu, ${ }^{32 b}$ E. Wulf,${ }^{34}$ R. Wunstorf,${ }^{42}$ B. M. Wynne, ${ }^{45}$ L. Xaplanteris, ${ }^{9}$ S. Xella, ${ }^{35}$ S. Xie, ${ }^{48}$ Y. Xie, ${ }^{32 \mathrm{a}}$ C. Xu, ${ }^{32 \mathrm{~b}}$ D. Xu, ${ }^{139}$ G. Xu,${ }^{32 \mathrm{a}}$ B. Yabsley, ${ }^{150}$ M. Yamada, ${ }^{66}$ A. Yamamoto ${ }^{66} \mathrm{~K}$. Yamamoto, ${ }^{64}$ S. Yamamoto, ${ }^{155}$ T. Yamamura, ${ }^{155}$ J. Yamaoka, ${ }^{44}$ T. Yamazaki, ${ }^{155}$ Y. Yamazaki, ${ }^{67}$ Z. Yan, ${ }^{21}$ H. Yang, ${ }^{87}$ U. K. Yang, ${ }^{82}$ Y. Yang, ${ }^{61}$ Y. Yang, ${ }^{32 \mathrm{a}}$ Z. Yang, ${ }^{146 a, 146 b}$ S. Yanush, ${ }^{91}$ W-M. Yao, ${ }^{14}$ Y. Yao, ${ }^{14}$ Y. Yasu, ${ }^{66}$ G. V. Ybeles Smit, ${ }^{130}$ J. Ye, ${ }^{39}$ S. Ye, ${ }^{24}$ M. Yilmaz,${ }^{3 c}$ R. Yoosoofmiya, ${ }^{123}$ K. Yorita, ${ }^{170}$ R. Yoshida,${ }^{5}$ C. Young, ${ }^{143}$ S. Youssef, ${ }^{21}$ D. Yu, ${ }^{24}$ J. Yu, ${ }^{7}$

J. Yu, ${ }^{32 c \text {,aa }}$ L. Yuan, ${ }^{32 a, b b}$ A. Yurkewicz, ${ }^{148}$ V. G. Zaets, ${ }^{128}$ R. Zaidan, ${ }^{63}$ A. M. Zaitsev, ${ }^{128}$ Z. Zajacova, ${ }^{29}$ Yo. K. Zalite, ${ }^{121}$ L. Zanello, ${ }^{132 a, 132 b}$ P. Zarzhitsky, ${ }^{39}$ A. Zaytsev, ${ }^{107}$ C. Zeitnitz, ${ }^{174}$ M. Zeller, ${ }^{175}$ P. F. Zema, ${ }^{29}$ 
A. Zemla, ${ }^{38}$ C. Zendler, ${ }^{20}$ A. V. Zenin, ${ }^{128}$ O. Zenin, ${ }^{128}$ T. Ženiš, ${ }^{144 a}$ Z. Zenonos, ${ }^{122 a, 122 b}$ S. Zenz, ${ }^{14}$ D. Zerwas, ${ }^{115}$ G. Zevi della Porta, ${ }^{57}$ Z. Zhan, ${ }^{32 \mathrm{~d}}$ D. Zhang, ${ }^{32 \mathrm{~b}}$ H. Zhang, ${ }^{88}$ J. Zhang, ${ }^{5}$ X. Zhang, ${ }^{32 \mathrm{~d}}$ Z. Zhang, ${ }^{115}$ L. Zhao, ${ }^{108}$ T. Zhao, ${ }^{138}$ Z. Zhao, ${ }^{32 b}$ A. Zhemchugov, ${ }^{65}$ S. Zheng, ${ }^{32 \mathrm{a}}$ J. Zhong, ${ }^{151, \mathrm{cc}}$ B. Zhou, ${ }^{87}$ N. Zhou, ${ }^{163}$ Y. Zhou, ${ }^{151}$ C. G. Zhu, ${ }^{32 \mathrm{~d}}$ H. Zhu ${ }^{41}$ J. Zhu ${ }^{87}$ Y. Zhu, ${ }^{172}$ X. Zhuang, ${ }^{98}$ V. Zhuravlov, ${ }^{99}$ D. Zieminska, ${ }^{61}$ R. Zimmermann, ${ }^{20}$ S. Zimmermann, ${ }^{20}$ S. Zimmermann, ${ }^{48}$ M. Ziolkowski, ${ }^{141}$ R. Zitoun, ${ }^{4}$ L. Živković,${ }^{34}$ V. V. Zmouchko, ${ }^{128, a}$ G. Zobernig, ${ }^{172}$ A. Zoccoli, ${ }^{19 a, 19 b}$ Y. Zolnierowski, ${ }^{4}$ A. Zsenei, ${ }^{29}$ M. zur Nedden, ${ }^{15}$ V. Zutshi, ${ }^{106}$ and L. Zwalinski ${ }^{29}$

\title{
(ATLAS Collaboration)
}

\author{
${ }^{1}$ University at Albany, Albany, New York, USA \\ ${ }^{2}$ Department of Physics, University of Alberta, Edmonton Alberta, Canada \\ ${ }^{3 a}$ Department of Physics, Ankara University, Ankara, Turkey \\ ${ }^{3 \mathrm{~b}}$ Department of Physics, Dumlupinar University, Kutahya, Turkey \\ ${ }^{3 c}$ Department of Physics, Gazi University, Ankara, Turkey \\ ${ }^{3 \mathrm{~d}}$ Division of Physics, TOBB University of Economics and Technology, Ankara, Turkey \\ ${ }^{3 e}$ Turkish Atomic Energy Authority, Ankara, Turkey \\ ${ }^{4} L A P P, C N R S / I N 2 P 3$ and Université de Savoie, Annecy-le-Vieux, France \\ ${ }^{5}$ High Energy Physics Division, Argonne National Laboratory, Argonne, Illinois, USA \\ ${ }^{6}$ Department of Physics, University of Arizona, Tucson, Arizona, USA \\ ${ }^{7}$ Department of Physics, The University of Texas at Arlington, Arlington, Texas, USA \\ ${ }^{8}$ Physics Department, University of Athens, Athens, Greece \\ ${ }^{9}$ Physics Department, National Technical University of Athens, Zografou, Greece \\ ${ }^{10}$ Institute of Physics, Azerbaijan Academy of Sciences, Baku, Azerbaijan \\ ${ }^{11}$ Institut de Física d'Altes Energies and Universitat Autònoma de Barcelona and ICREA, Barcelona, Spain \\ ${ }^{12 a}$ Institute of Physics, University of Belgrade, Belgrade, Serbia \\ ${ }^{12 \mathrm{~b}}$ Vinca Institute of Nuclear Sciences, Belgrade, Serbia \\ ${ }^{13}$ Department for Physics and Technology, University of Bergen, Bergen, Norway \\ ${ }^{14}$ Physics Division, Lawrence Berkeley National Laboratory and University of California, Berkeley, California, USA \\ ${ }^{15}$ Department of Physics, Humboldt University, Berlin, Germany \\ ${ }^{16}$ Albert Einstein Center for Fundamental Physics and Laboratory for High Energy Physics, University of Bern, Bern, Switzerland \\ ${ }^{17}$ School of Physics and Astronomy, University of Birmingham, Birmingham, United Kingdom \\ ${ }^{18 a}$ Department of Physics, Bogazici University, Istanbul, Turkey \\ ${ }^{18 \mathrm{~b}}$ Division of Physics, Dogus University, Istanbul, Turkey \\ ${ }^{18 c}$ Department of Physics Engineering, Gaziantep University, Gaziantep, Turkey \\ ${ }^{18 \mathrm{~d}}$ Department of Physics, Istanbul Technical University, Istanbul, Turkey \\ ${ }^{19 a}$ INFN Sezione di Bologna, Italy \\ ${ }^{19 \mathrm{~b}}$ Dipartimento di Fisica, Università di Bologna, Bologna, Italy \\ ${ }^{20}$ Physikalisches Institut, University of Bonn, Bonn, Germany \\ ${ }^{21}$ Department of Physics, Boston University, Boston, Massachusetts, USA \\ ${ }^{22}$ Department of Physics, Brandeis University, Waltham, Massachusetts, USA \\ ${ }^{23 a}$ Universidade Federal do Rio De Janeiro COPPE/EE/IF, Rio de Janeiro, Brazil \\ ${ }^{23 \mathrm{~b}}$ Instituto de Fisica, Universidade de Sao Paulo, Sao Paulo, Brazil \\ ${ }^{24}$ Physics Department, Brookhaven National Laboratory, Upton, New York, USA \\ ${ }^{25 \mathrm{a}}$ National Institute of Physics and Nuclear Engineering, Bucharest, Romania \\ ${ }^{25 b}$ University Politehnica Bucharest, Bucharest, Romania \\ ${ }^{25 c}$ West University in Timisoara, Timisoara, Romania \\ ${ }^{26}$ Departamento de Física, Universidad de Buenos Aires, Buenos Aires, Argentina \\ ${ }^{27}$ Cavendish Laboratory, University of Cambridge, Cambridge, United Kingdom \\ ${ }^{28}$ Department of Physics, Carleton University, Ottawa Ontario, Canada \\ ${ }^{29}$ CERN, Geneva, Switzerland \\ ${ }^{30}$ Enrico Fermi Institute, University of Chicago, Chicago, Illinois, USA \\ ${ }^{31 a}$ Departamento de Fisica, Pontificia Universidad Católica de Chile, Santiago, Chile \\ ${ }^{31 \mathrm{~b}}$ Departamento de Física, Universidad Técnica Federico Santa María, Valparaíso, Chile \\ ${ }^{32 a}$ Institute of High Energy Physics, Chinese Academy of Sciences, Beijing, China \\ ${ }^{32 \mathrm{~b}}$ Department of Modern Physics, University of Science and Technology of China, Anhui, China \\ ${ }^{32 \mathrm{c}}$ Department of Physics, Nanjing University, Jiangsu, China

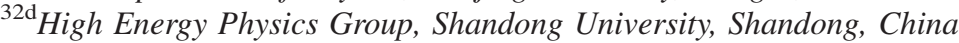 \\ ${ }^{33}$ Laboratoire de Physique Corpusculaire, Clermont Université and Université Blaise Pascal and CNRS/IN2P3, \\ Aubiere Cedex, France
}




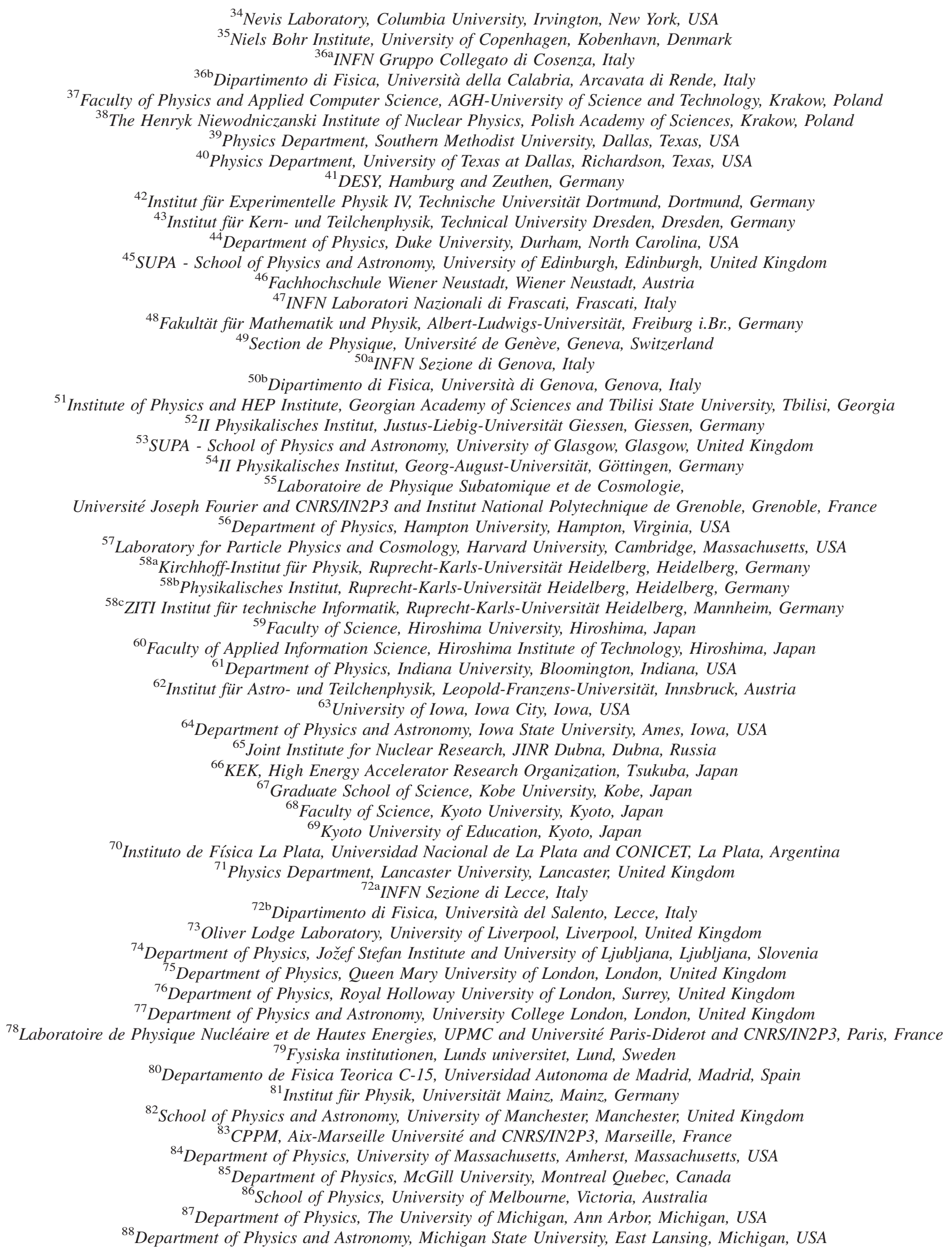




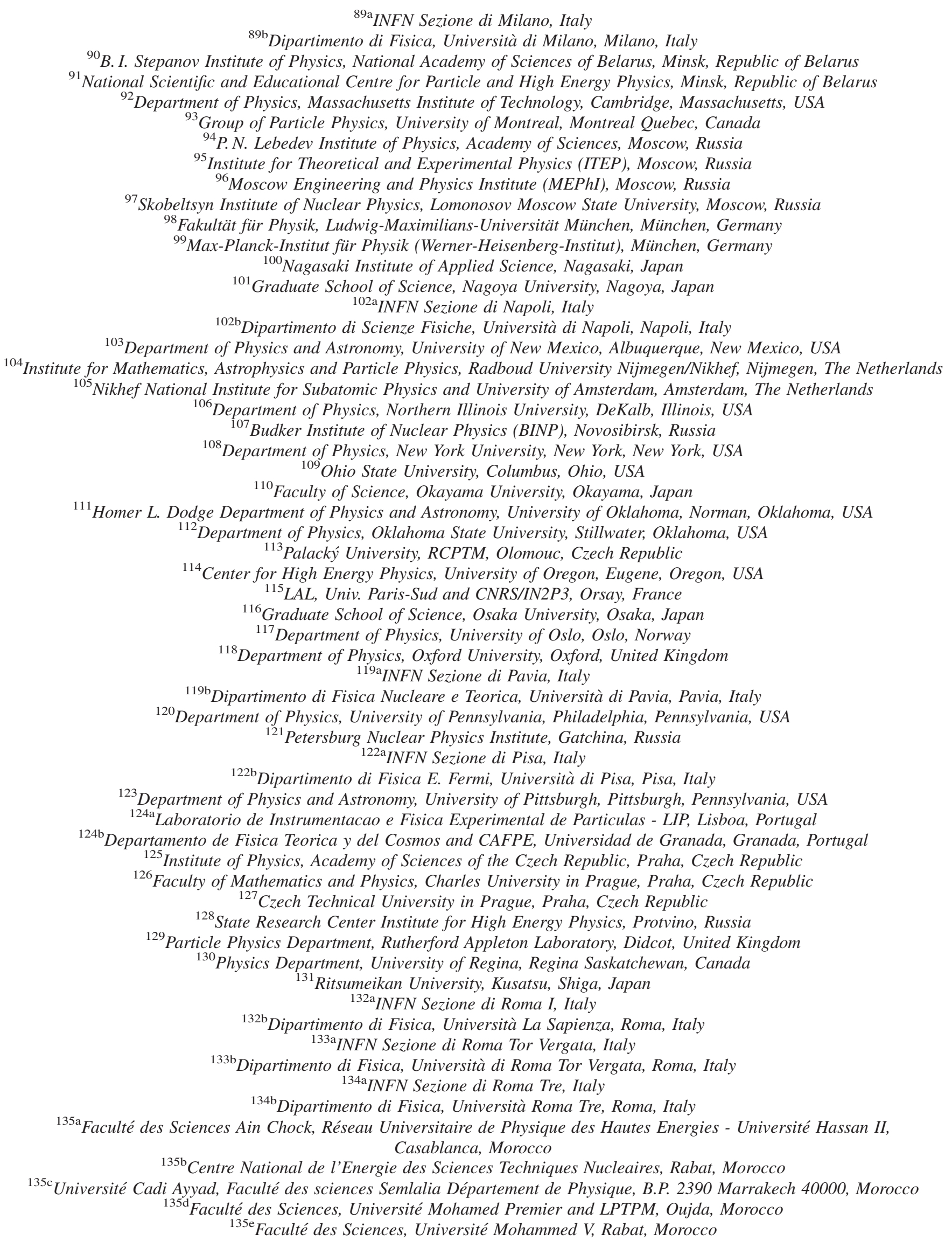


${ }^{136}$ DSM/IRFU (Institut de Recherches sur les Lois Fondamentales de l'Univers),

CEA Saclay (Commissariat a l'Energie Atomique), Gif-sur-Yvette, France

${ }^{137}$ Santa Cruz. Institute for Particle Physics, University of California Santa Cruz, Santa Cruz, California, USA

${ }^{138}$ Department of Physics, University of Washington, Seattle, Washington, USA

${ }^{139}$ Department of Physics and Astronomy, University of Sheffield, Sheffield, United Kingdom

${ }^{140}$ Department of Physics, Shinshu University, Nagano, Japan

${ }^{141}$ Fachbereich Physik, Universität Siegen, Siegen, Germany

${ }^{142}$ Department of Physics, Simon Fraser University, Burnaby British Columbia, Canada

${ }^{143}$ SLAC National Accelerator Laboratory, Stanford, California, USA

${ }^{144 a}$ Faculty of Mathematics, Physics and Informatics, Comenius University, Bratislava, Slovak Republic

${ }^{144 b}$ Department of Subnuclear Physics, Institute of Experimental Physics of the Slovak Academy of Sciences, Kosice, Slovak Republic

${ }^{145 a}$ Department of Physics, University of Johannesburg, Johannesburg, South Africa

${ }^{145 \mathrm{~b}}$ School of Physics, University of the Witwatersrand, Johannesburg, South Africa

${ }^{146 a}$ Department of Physics, Stockholm University, Sweden

${ }^{146 \mathrm{~b}}$ The Oskar Klein Centre, Stockholm, Sweden

${ }^{147}$ Physics Department, Royal Institute of Technology, Stockholm, Sweden

${ }^{148}$ Department of Physics and Astronomy, Stony Brook University, Stony Brook, New York, USA

${ }^{149}$ Department of Physics and Astronomy, University of Sussex, Brighton, United Kingdom

${ }^{150}$ School of Physics, University of Sydney, Sydney, Australia

${ }^{151}$ Institute of Physics, Academia Sinica, Taipei, Taiwan

${ }^{152}$ Department of Physics, Technion: Israel Institute of Technology, Haifa, Israel

${ }^{153}$ Raymond and Beverly Sackler School of Physics and Astronomy, Tel Aviv University, Tel Aviv, Israel

${ }^{154}$ Department of Physics, Aristotle University of Thessaloniki, Thessaloniki, Greece

${ }^{155}$ International Center for Elementary Particle Physics and Department of Physics, The University of Tokyo, Tokyo, Japan

${ }^{156}$ Graduate School of Science and Technology, Tokyo Metropolitan University, Tokyo, Japan

${ }^{157}$ Department of Physics, Tokyo Institute of Technology, Tokyo, Japan

${ }^{158}$ Department of Physics, University of Toronto, Toronto Ontario, Canada

${ }^{159 a}$ TRIUMF, Vancouver British Columbia, Canada

${ }^{159 \mathrm{~b}}$ Department of Physics and Astronomy, York University, Toronto Ontario, Canada

${ }^{160}$ Institute of Pure and Applied Sciences, University of Tsukuba, Ibaraki, Japan

${ }^{161}$ Science and Technology Center, Tufts University, Medford, Massachusetts, USA

${ }^{162}$ Centro de Investigaciones, Universidad Antonio Narino, Bogota, Colombia

${ }^{163}$ Department of Physics and Astronomy, University of California Irvine, Irvine, California, USA

${ }^{164 a}$ INFN Gruppo Collegato di Udine, Italy

${ }^{164 \mathrm{~b}}$ ICTP, Trieste, Italy

${ }^{164 c}$ Dipartimento di Fisica, Università di Udine, Udine, Italy

${ }^{165}$ Department of Physics, University of Illinois, Urbana, Illinois, USA

${ }^{166}$ Department of Physics and Astronomy, University of Uppsala, Uppsala, Sweden

${ }^{167}$ Instituto de Física Corpuscular (IFIC) and Departamento de Física Atómica,

Molecular y Nuclear and Departamento de Ingenierá Electrónica and Instituto de Microelectrónica de Barcelona (IMB-CNM),

University of Valencia and CSIC, Valencia, Spain

${ }^{168}$ Department of Physics, University of British Columbia, Vancouver British Columbia, Canada

${ }^{169}$ Department of Physics and Astronomy, University of Victoria, Victoria British Columbia, Canada

${ }^{170}$ Waseda University, Tokyo, Japan

${ }^{171}$ Department of Particle Physics, The Weizmann Institute of Science, Rehovot, Israel

${ }^{172}$ Department of Physics, University of Wisconsin, Madison, Wisconsin, USA

${ }^{173}$ Fakultät für Physik und Astronomie, Julius-Maximilians-Universität, Würzburg, Germany

${ }^{174}$ Fachbereich C Physik, Bergische Universität Wuppertal, Wuppertal, Germany

${ }^{175}$ Department of Physics, Yale University, New Haven, Connecticut, USA

${ }^{176}$ Yerevan Physics Institute, Yerevan, Armenia

${ }^{177}$ Domaine scientifique de la Doua, Centre de Calcul CNRS/IN2P3, Villeurbanne Cedex, France

${ }^{\mathrm{a}}$ Deceased.

${ }^{\mathrm{b}}$ Also at Laboratorio de Instrumentacao e Fisica Experimental de Particulas - LIP, Lisboa, Portugal.

${ }^{\mathrm{c}}$ Also at Faculdade de Ciencias and CFNUL, Universidade de Lisboa, Lisboa, Portugal.

${ }^{\mathrm{d} A 1 s o}$ at CPPM, Aix-Marseille Université and CNRS/IN2P3, Marseille, France.

${ }^{\mathrm{e}}$ Also at TRIUMF, Vancouver BC, Canada.

${ }^{\mathrm{f}}$ Also at Department of Physics, California State University, Fresno, CA, USA.

${ }^{\mathrm{g}}$ Also at Faculty of Physics and Applied Computer Science, AGH-University of Science and Technology, Krakow, Poland.

${ }^{\mathrm{h}}$ Also at Department of Physics, University of Coimbra, Coimbra, Portugal. 
${ }^{\mathrm{i}}$ Also at Università di Napoli Parthenope, Napoli, Italy.

${ }^{j}$ Also at Institute of Particle Physics (IPP), Canada.

${ }^{k}$ Also at Louisiana Tech University, Ruston, LA, USA.

${ }^{1}$ Also at Group of Particle Physics, University of Montreal, Montreal QC, Canada.

${ }^{\mathrm{m}}$ Also at Institute of Physics, Azerbaijan Academy of Sciences, Baku, Azerbaijan.

${ }^{\mathrm{n}}$ Also at Institut für Experimentalphysik, Universität Hamburg, Hamburg, Germany.

${ }^{\circ}$ Also at Manhattan College, New York, NY, USA.

${ }^{\mathrm{p}}$ Also at School of Physics and Engineering, Sun Yat-sen University, Guanzhou, China.

${ }^{\mathrm{q}}$ Also at Academia Sinica Grid Computing, Institute of Physics, Academia Sinica, Taipei, Taiwan.

${ }^{\mathrm{r}}$ Also at High Energy Physics Group, Shandong University, Shandong, China.

${ }^{\text {s} A l s o ~ a t ~ C a l i f o r n i a ~ I n s t i t u t e ~ o f ~ T e c h n o l o g y, ~ P a s a d e n a, ~ C A, ~ U S A . ~}$

${ }^{\mathrm{t} A l s o}$ at Particle Physics Department, Rutherford Appleton Laboratory, Didcot, United Kingdom.

uAlso at Section de Physique, Université de Genève, Geneva, Switzerland.

${ }^{v}$ Also at Departamento de Fisica, Universidade de Minho, Braga, Portugal.

${ }^{w}$ Also at Department of Physics and Astronomy, University of South Carolina, Columbia, SC, USA.

${ }^{\mathrm{x}}$ Also at KFKI Research Institute for Particle and Nuclear Physics, Budapest, Hungary.

${ }^{\mathrm{y}}$ Also at Institute of Physics, Jagiellonian University, Krakow, Poland.

${ }^{\mathrm{z}}$ Also at Department of Physics, Oxford University, Oxford, United Kingdom.

${ }^{\text {aa }}$ Also at DSM/IRFU (Institut de Recherches sur les Lois Fondamentales de l'Univers), CEA Saclay (Commissariat a 1'Energie Atomique), Gif-sur-Yvette, France.

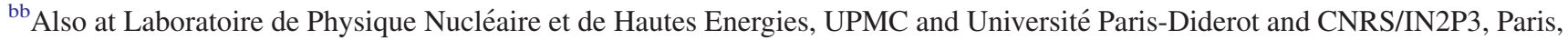
France.

${ }^{\mathrm{cc}}$ Also at Department of Physics, Nanjing University, Jiangsu, China. 